\title{
Icariin, an Up-and-Coming Bioactive Compound Against Neurological Diseases: Network Pharmacology-Based Study and Literature Review
}

\begin{abstract}
Shuangqiu Wang, ${ }^{\mathrm{I}-3, *}$ Jiarui Ma, ${ }^{4, *}$ Yanqi Zeng, ${ }^{5, *}$ Guowei Zhou, iD ${ }^{6}$

Yuxuan Wang, ${ }^{1-3}$ Wenjuan Zhou, ${ }^{5}$ Xiaohe Sun, ${ }^{5}$ Minghua Wu (iD) 1,5

'Department of Neurology, Jiangsu Province Hospital of Chinese Medicine, Affiliated Hospital of Nanjing University of Chinese Medicine, Nanjing, 210029, Jiangsu, People's Republic of China; ${ }^{2}$ Key Laboratory of Acupuncture and Medicine Research of Ministry of Education, Nanjing University of Chinese Medicine, Nanjing, 2 10046, People's Republic of China; ${ }^{3}$ State Key Laboratory Cultivation Base for TCM Quality and Efficacy, Nanjing University of Chinese Medicine, Nanjing, 2 10046, Jiangsu, People's Republic of China; ${ }^{4}$ Provincial Key Laboratory of Drug Target and Drug for

Degenerative Disease, School of Medicine and Holistic Integrative Medicine, Nanjing University of Chinese Medicine, Nanjing, 2 10046, Jiangsu, People's Republic of China; ${ }^{5}$ First Clinical Medical School, Jiangsu Province Hospital of Chinese Medicine, Affiliated Hospital of Nanjing University of Chinese Medicine, Nanjing, 2 I0046, Jiangsu, People's Republic of China; ${ }^{6}$ Department of General Surgery, Jiangsu Province Hospital of Chinese Medicine, Affiliated Hospital of Nanjing University of Chinese Medicine, Nanjing, 210029, Jiangsu, People's Republic of China
\end{abstract}

*These authors contributed equally to this work

Correspondence: Minghua Wu

Department of Neurology, Jiangsu

Provincial Hospital of Chinese Medicine,

The Affiliated Hospital of Nanjing

University of Chinese Medicine, No. 155

Hanzhong Road, Qinhuai District,

Nanjing, Jiangsu, People's Republic of

China

Tel +86- $139517867 \mid 9$

$\mathrm{Fax}+8625866|7| 4 \mid$

Email mhuawu@163.com

\begin{abstract}
Icariin is a biologically active substance in Epimedii herba that is used for the treatment of neurologic disorders. However, a comprehensive analysis of the molecular mechanisms of icariin is lacking. In this review, we present a brief history of the use of icariin for medicinal purposes; describe the active chemical components of Epimedii herba; and examine the evidence from experimental studies that have uncovered molecular targets of icariin in different diseases. We also constructed a protein-protein interaction network and carried out Gene Ontology and Kyoto Encyclopedia of Genes and Genomes functional enrichment analyses to predict the therapeutic actions of icariin in nervous system diseases including Alzheimer disease, Parkinson disease, ischemic stroke, depressive disorder, multiple sclerosis, glioblastoma, and hereditary spastic paraplegias. The results of our analyses can guide future studies on the application of icariin to the treatment of neurologic disorders.
\end{abstract}

Keywords: Epimedii herba, icariin, network pharmacology, literature review, nervous system diseases

\section{Introduction}

Neurologic disorders are the leading cause of disability and the second leading cause of death worldwide. ${ }^{1}$ Degenerative, inflammatory, infectious, vascular, and neoplastic disorders of the central nervous system (CNS) are among the most severe diseases in humans; and cerebrovascular diseases such as stroke are a considerable burden and challenge for individuals and for society. Unhealthy lifestyle habits including alcoholism, smoking, and a high-fat diet, as well as other factors such as aging, psychosocial stress, and environmental pollution contribute to the occurrence and development of stroke. ${ }^{2}$ Dementia accounts for $10 \%$ of neurologic disorders. ${ }^{3}$ Alzheimer disease (AD), the most common form of dementia, is a major strain on healthcare systems because of its chronic and debilitating nature. ${ }^{4}$ Different medications are used to treat neurologic diseases such as stroke and AD. For example, alteplase, nimodipine, and aspirin are conventional therapeutics for cerebrovascular diseases. ${ }^{5,6}$ Alteplase, a recombinant tissue plasminogen activator, has demonstrated benefits in the treatment of severe ischemic stroke; however, it can also delay reperfusion and increases the risk of intracranial hemorrhage. ${ }^{7}$ Rivastigmine is often used to alleviate the symptoms of AD but its side effects such as nausea, diarrhea, vomiting, and dizziness limit its clinical use. ${ }^{8}$ 
Recently, active compounds of herbal medicines have attracted interest in the medical community because of their pharmacologic activity combined with low toxicity. For example, the sesquiterpene lactone artemisinin is a natural product used to treat malaria. ${ }^{9}$ Herbal medicines have also shown benefits in the treatment of nervous system diseases. As an ancient natural aphrodisiac, Epimedii herba is commonly used in Chinese and Korean traditional medicine to treat nocturnal emissions, impotence, limb weakness, muscle contracture, lethargy, and headache. ${ }^{10,11}$ Natural products typically act via multiple targets and pathways. ${ }^{12}$ Icariin, the main chemical component of Epimedii herba is transported in the circulation and can penetrate the blood-brain barrier (BBB) to exert effects on the CNS. ${ }^{13-17}$ However, the pharmacologic properties of icariin and the molecular basis for its effects on nervous system diseases are not fully understood.

Network pharmacology is a relative novel approach for systematically and comprehensively investigating the mechanism of action of drugs ${ }^{18,19}$ that can be applied to natural products including icariin. ${ }^{20}$ The computational power of this approach can also facilitate the design of studies to experimentally validate the role of icariin in various diseases in a more efficient manner than by trial and error. ${ }^{21}$ In this review, we present a brief history of the use of icariin for medicinal purposes; describe the active chemical components of Epimedii herba; and examine experimental evidence from studies that have uncovered the molecular targets of icariin in different CNS diseases. We also carried out functional enrichment analyses to predict the mechanisms of action icariin in the treatment of these diseases. The results of these analyses, and the evidence from the literature presented herein, can guide future studies on the application of icariin to the treatment of neurologic disorders.

\section{History}

\section{Epimedii herba}

Epimedii herba is the dried leaf of epimedium - an herbaceous plant belonging to the Berberidaceae family ${ }^{22}$ - and is commonly referred to as horny goat weed, Xian-Ling-Pi, Gang-Qian, and San-Zhi-Jiu-Ye-Cao. Epimedii herba is widely distributed across eastern, southern, and central Asia and Europe $\mathrm{e}^{23}$ and has an over 2000-year history of clinical application in countries such as China, South Korea, and Japan. Epimedii herba was initially documented in Sheng Nong Ben Cao Jing, the oldest classical text on medicinal plants in China. Ben Cao Gang Mu, another important compendium of traditional Chinese medicine, describes Epimedii herba as pungent, cold, and nontoxic. Since 1963, Epimedii herba has been officially listed in the pharmacopeia of the People's Republic of China as a treatment for chronic conditions such as hemiparesis and erectile dysfunction. ${ }^{9,24}$ The medicinal benefits of Epimedii herba include strengthening the body, improving fertility, and relieving stress and fatigue. Practitioners of traditional Chinese medicine use Epimedii herba as a remedy for kidney-Yang deficiency syndrome, which is characterized by soreness or a cold sensation in the loins and knee, impotence, seminal emission, female sterility, difficulty in urination, or general edema. According to the Chromatographic Fingerprint Analysis of Herbal Medicines, Epimedii herba can be used to treat neurasthenia, climacteric hypertension, chronic bronchitis, viral myocarditis, and leucopenia. ${ }^{25}$ More recently, the therapeutic effects of Epimedii herba on the reproductive ${ }^{26}$ and skeletal ${ }^{27}$ system have been experimentally validated. Epimedii herba also has demonstrated neuroplasticity-promoting ${ }^{10}$ and antioxidatnt ${ }^{28}$ effects.

\section{Icariin}

Icariin is a well-characterized component of Epimedii herba with multiple potent biological activities. Icariin was first isolated and identified in 1990 from the oral liquid of Luohan Jindan by column partition chromatography-thin layer chromatography-ultraviolet spectroscopy. ${ }^{29}$ A highspeed countercurrent chromatography technique was later used to extract icariin from epimedium with $>98 \%$ purity. ${ }^{30}$ Microwave pretreatment of raw materials significantly improved the efficiency of icariin extraction. ${ }^{31}$ Icariin was reported to function as a regulator of adaptive immunity in $1995,{ }^{32}$ and subsequent studies have revealed antihepatotoxic ${ }^{33}$ and antioxidant ${ }^{34}$ activities as well as the ability to stimulate corticosterone production ${ }^{35}$ and promote neurite outgrowth. ${ }^{36}$ Icariin has pharmacologic effects on the immune, ${ }^{37}$ skeletal $^{38}$ and reproductive ${ }^{39}$ systems. Over the past few years, an increasing number of studies have focused on the protective effects of icariin in the nervous system diseases.

\section{Active Chemical Compounds of Epimedii herba}

\section{Flavonoids}

A total of 53 flavonoids have been identified in Epimedii herba including flavones, biflavonoids, flavanones, and 
flavonoid glycosides, such as baohuoside I, 5,7,4' trihydroxy-8,3'-diprenylflavone, ginkgetin, robinetin, apigenin, luteolin, hyperin, and icariin. Some flavonoids in Epimedii herba have demonstrated medicinal benefits. ${ }^{40}$ Baohuoside I was shown to alleviate cognitive dysfunction and exert antiosteoporotic, and antitumor effects. ${ }^{41}$ Ginkgetin is a natural biflavonoid with anti-inflammatory, antiviral, and antitumor properties. ${ }^{42}$ Icariin, a flavonoid glycoside, is the main bioactive ingredient of Epimedii herba and is a promising therapeutic agent for the treatment of various disorders because of its antiapoptotic, anti-inflammatory, and antioxidant activities. ${ }^{43,44}$

\section{Terpenoids}

Fifteen terpenoids have been isolated from Epimedii herba that are classified as monoterpenes, sesquiterpenes, and triterpenes. Terpenoids exert beneficial effects on bone metabolism and have anti-inflammatory, neuroprotective, and antioxidant functions. ${ }^{45,46}$ Oleanolic acid (OA), a pentacyclic triterpenoid, has pharmacologic effects in osteoporosis and neurodegenerative disease. For example, OA was shown to induce the differentiation of bone marrowderived mesenchymal stem cells into osteoblasts by regulating the cell cycle and metabolism ${ }^{47}$ or by inhibiting Notch signaling, ${ }^{48}$ and regulated calcium balance by promoting calcium entry across the brush border membrane ${ }^{49} \mathrm{OA}$ was also found to exert a neuroprotective effect by activating nuclear factor erythroid 2-related factor (Nrf)2 and inhibiting the expression of nitric oxide synthase in the hypoxic brain. ${ }^{50}$ Given its modulatory effects on endogenous antioxidants and mitochondrial function, $\mathrm{OA}$ is considered as a promising agent for the treatment of cerebral ischemia. ${ }^{51}$

\section{Other Compounds}

Several phytochemicals, such as steroids, acids, lignans, alkaloids, and anthraquinones have been isolated from Epimedii herba. Treatment with the steroid sitosterol significantly reduced the immobility time of rats in the forced swim and tail suspension tests, indicating that sitosterol has an antidepressant effect that may involve increasing 5-hydroxytryptamine and norepinephrine levels in the CNS. ${ }^{52}$ Lauric acid can stimulates the production of ketone bodies by astrocytes, which exert a neuroprotective effect on adjacent neurons. ${ }^{53}$ Emodin is a naturally occurring anthraquinone derivative that was reported to confer neuroprotection in $\mathrm{AD}$ and epilepsy and alleviate ischemia-reperfusion brain injury and glutamate-induced neuronal damage. ${ }^{54}$ The alkaloid magnoflorine increased the latency index of mice in the passive avoidance test by inhibiting acetylcholinesterase, suggesting a role in improving short-term memory. Thus, magnoflorine is a promising candidate drug for the treatment of diseases associated with memory deficits such as dementia or $\mathrm{AD} .{ }^{55} \mathrm{In}$ a rat model of global brain ischemia-reperfusion p-tyrosol prevented neuronal loss in the hippocampus. ${ }^{56}$ lsoliquiritigenin suppressed the inflammatory response after traumatic brain injury by inhibiting the phosphoinositide 3-kinase (PI3K)/protein kinase B (AKT)/glycogen synthase kinase-3 beta (GSK-3 $\beta$ )/nuclear factor kappa B (NF-kB) signaling axis. ${ }^{57}$ In summary, the evidence to date indicates that compounds found in Epimedii herba can improve neurologic function and mitigate nervous system damage although the molecular mechanisms underlying these effects are not well understood.

\section{Network Pharmacology Analysis of Icariin Screening of Potential Targets}

In order to elucidate the molecular basis for the pharmacologic effects of icariin based on the existing evidence, we constructed a database of molecular targets in neurologic diseases including AD (disease ID: C0002395, $\mathrm{n}=1981$ ), Parkinson disease (PD; disease ID: C0030567, $\mathrm{n}=1063$ ), ischemic stroke (disease ID: C0948008, $\mathrm{n}=393$ ), depressive disorder (disease ID: C0011581, $\mathrm{n}=740$ ), multiple sclerosis (MS; disease ID: C0026769, n=1105), glioblastoma (disease ID: C0017636, $\mathrm{n}=1936$ ), and spastic paraplegias (disease ID: C0037772, $n=312$ ) using the DisGeNET database v6.0 (https://www.disgenet.org/). ${ }^{58,59}$ In a previous report, ${ }^{59} 219$ targets of icariin were predicted using PharmMapper (http://lilab-ecust.cn/pharmmapper/), Drug Repositioning and Adverse Reaction via ChemicalProtein Interactome (https://cpi.bio-X.cn/drar/), TargetNet (http://targetnet.scbdd.com/), and ChemMapper (http:// lilab-ecust.cn/chemmapper/) after eliminating duplicates (Figure 1). The intersection of each disease with icariin identified 72 targets in $\mathrm{AD}, 33$ in $\mathrm{PD}, 15$ in ischemic stroke, 32 in depressive disorder, 29 in MS, 73 in glioblastoma and 2 in spastic paraplegia (Supplementary Table S1); all of these were standardized according to their gene names by searching the UniProtKB (https://www.UniProt. org/) database with "Homo sapiens" as the species. ${ }^{60}$ Compound-target interactions were determined using Cytoscape v3.7.2 software (https://cytoscape.org/) and are shown in Figure 1. 


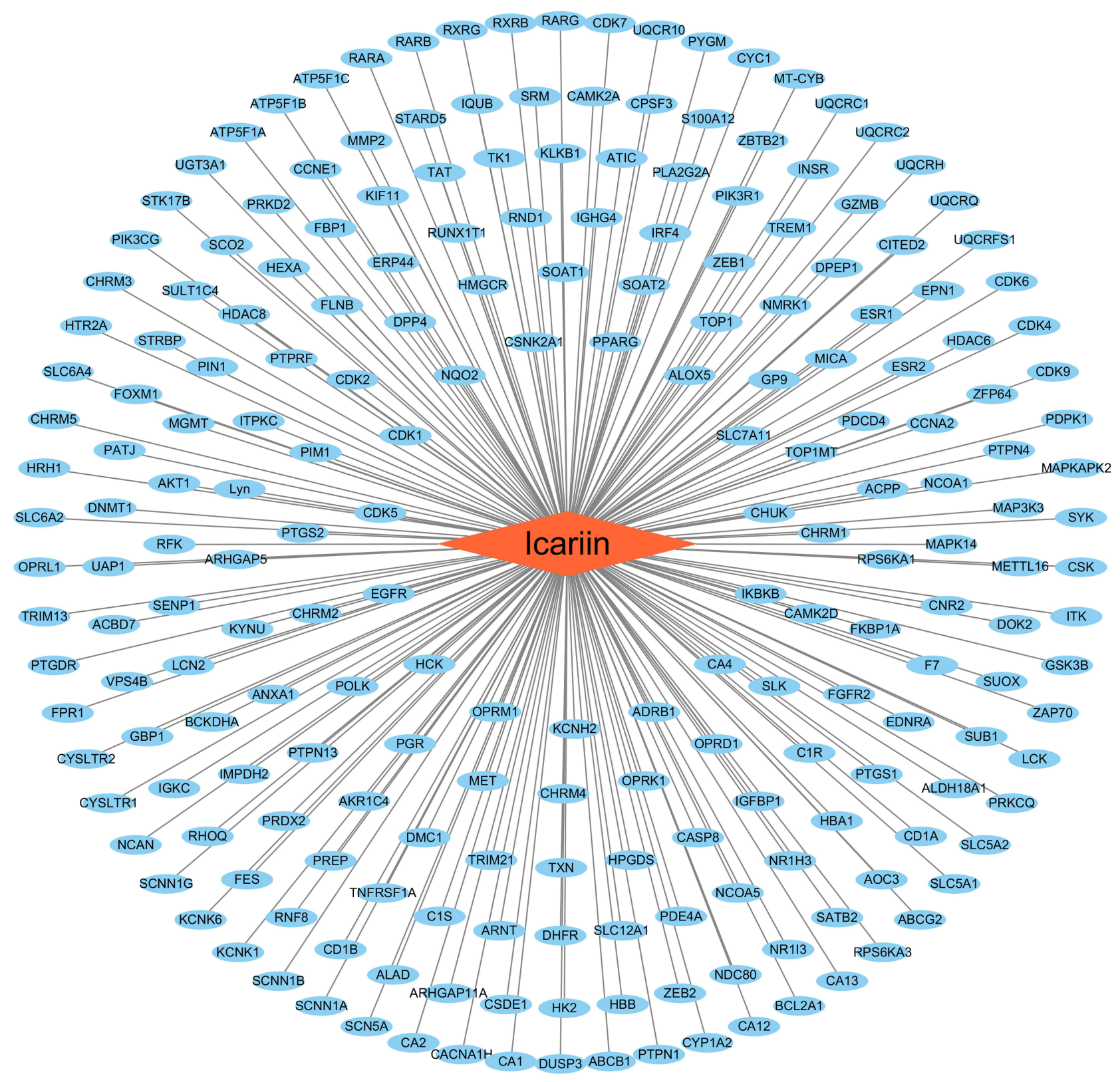

Figure I Compound-target interaction. Circular nodes represented targets and rhombic node represented icariin. Edges represented interactions between icariin and targets.

\section{Constructing the Protein-Protein Interaction (PPI) Network}

As proteins involved in biochemical processes form macromolecular complexes to execute biological functions, exploring the interaction of icariin with different proteins and their networks is important for characterizing its pharmacologic activities. We constructed a PPI network as described in our previous work. ${ }^{19}$ All 219 putative targets of icariin were entered into the Search Tool for the Retrieval of Interacting Genes/Proteins (v
11.0 (https://string-db.org/) to obtain relevant information on protein interactions, with the genes and interactions represented as network nodes and lines, respectively. ${ }^{61}$ The minimum score was set to the highest confidence value of 0.9 ; and proteins that were not connected to any others in the network were removed. We obtained a PPI network containing 216 nodes (representing active proteins) and 364 edges (representing the interactions between active proteins and other proteins) (Figure 2B). The average node degree was 3.37 and the 


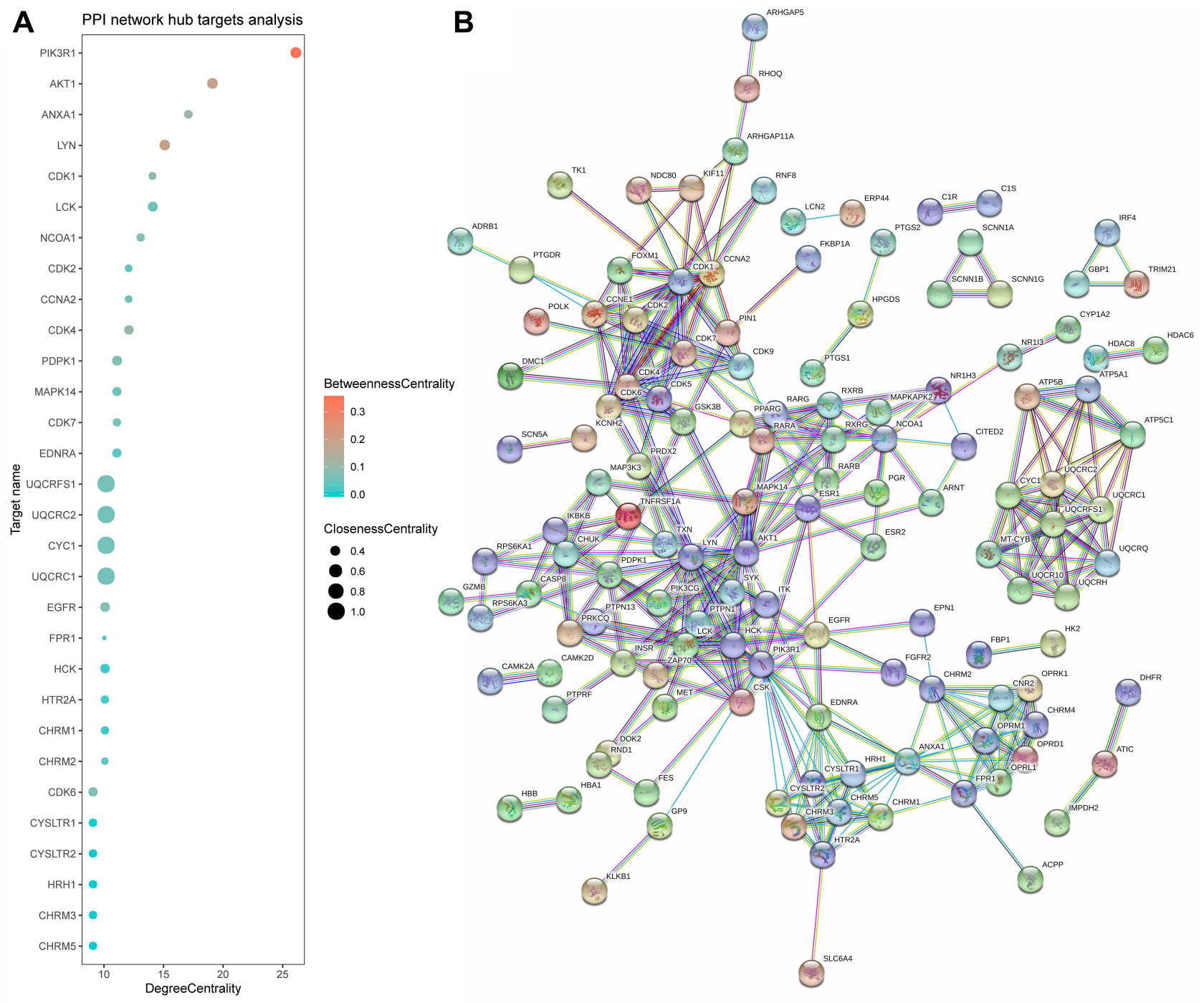

Figure 2 The PPI network for icariin and hub target analysis. (A) The centrality of targets was evaluated according to the degree centrality, betweenness centrality, and closeness centrality, which exhibited variation in terms of the $\mathbf{x}$-axis values, the colors of nodes, and the sizes of nodes. (B) The nodes indicated proteins, and edges represented protein-protein associations. The cyan edge indicated that the interaction was from a curated database, and the purple edge was experimentally determined. The green, red, and blue edges represented the interactions that were judged from the gene neighborhood, fusions, and cooccurrence, respectively.

average betweenness centrality was 0.435 . Based on a critical degree value $\geq 9,30$ targets were selected as hubs in the network (Figure 2A). The value of hub proteins was assessed based on degree, betweenness, and closeness centrality. The role of these targets in different diseases is discussed in detail below.

\section{Gene Ontology (GO) Analysis}

$\mathrm{GO}$ is an international standardized system for classifying gene function in 3 categories-namely, cellular component, molecular function, and biological process. ${ }^{62}$ The biological process category best reflects changes in the biological functions of the body. We first mapped all 219 targets of icariin to the GO biological process ontology in the GO database (http://www.geneontology.org/; Figure 3, and Supplementary Table S3). Gene numbers were calculated for every GO term, and the significance of enrichment relative to the background genome was evaluated with the hypergeometric test and subjected to false discovery rate (FDR) correction. FDR $\leq 0.05$ was set as the threshold for significant enrichment. The data were collected using the ClueGO and CluePedia Cytoscape plug-ins. ${ }^{63-65}$

The effects of icariin on biological processes were mainly related to the following functional groups: purine-containing compound metabolic process, cellular response to oxygen-containing compound, cellular response to reactive oxygen species (ROS), adenylate 
cyclase-inhibiting $\mathrm{G}$ protein-coupled receptor signaling pathway, regulation of organ growth, response to drug, and peptidyl-serine phosphorylation (Figure 3C). We observed that terms related to the regulation of nervous system function showed a higher level of enrichment; these included sensory perception of pain, opioid receptor pathway, opioid receptor activity, serotonin receptor signaling pathway, and G protein-coupled serotonin receptor signaling pathway. Additionally, there were several biological processes related to lipid metabolism, response to ROS, apoptosis-related signaling, regulation of immune cells, and cell cycle (Figure 3A and B). These results indicate that icariin acts on genes that are involved in nervous system function.

To clarify the effects of icariin on angiogenesis, we identified significantly enriched GO terms related to this process; ${ }^{66}$ the terms were depicted in visual form using R v3.6.1 software (http://www.r-project.org) with the clusterProfiler and ggplot2 packages (Figure 4).

\section{Kyoto Encyclopedia of Genes and Genomes (KEGG) Pathway Analysis}

KEGG pathway analysis (http://www.genome.jp/kegg/) can provide additional insight into the biological functions of genes. ${ }^{67}$ The KEGG pathway analysis of icariin targets revealed significant enrichment of metabolic or signal transduction pathways; the calculated p-values were subjected to FDR correction based on a threshold value of $p \leq 0.05$, and the data were collected using the ClueGO and CluePedia plugins. ${ }^{68,69}$ Target genes that were common to both the disease and icariin were also subjected to KEGG pathway analysis. No results were obtained for ischemic stroke and MS because of the limited number of common targets and stringent criterion for significance. The KEGG pathway enrichment results of $\mathrm{AD}$ and glioblastoma are shown in Figures 5 and 6 , respectively, with a filtering cutoff of $\geq 40$; results that are not shown in the figures can be found in Supplementary Table S2. The clusterProfiler package of R software available on Bioconductor (https://www. bioconductor.org/) was used to generate KEGG annotation graphs (Figure 6A). ${ }^{70,71}$

\section{Therapeutic Mechanisms of Icariin in Nervous System Diseases: Literature Review and Network Pharmacology Analysis \\ Potential Targets of Icariin in Neurologic Diseases}

Some targets of icariin were found to play a key role in the pathogenesis or treatment of nervous system diseases. For example, icariin may regulate GSK-3 $\beta$, a constitutively active serine/threonine-protein kinase that has been linked to the pathophysiology of $\mathrm{AD}, \mathrm{PD}$, and mood disorders (eg, depressive disorder) ${ }^{72-74}$ and was identified as a hub in the PPI network of icariin (Figure 7). Abnormal activation of GSK-3 $\beta$ has been demonstrated to accelerate the AD pathology process in AD patients. ${ }^{75}$ Amyloid (A) $\beta$ and amyloid precursor protein (APP) as well as hyperphosphorylated tau protein are involved in $\mathrm{AD}$ pathogenesis. ${ }^{76}$ GSK- $3 \beta$ regulates $A \beta$ production by interfering with APP cleavage, ${ }^{77}$ while GSK-3 $\beta$ inhibition decreased $\beta$-secretase (BACE1)-mediated cleavage of APP via a mechanism involving nuclear factor (NF) $\mathrm{\kappa B}$ signaling, thereby alleviating $A \beta$ pathology. ${ }^{78}$ GSK-3 $\beta$ controls numerous signaling pathways in the brain that promote tau hyperphosphorylation and neuronal degeneration, and interfere with normal synaptic plasticity. ${ }^{79}$ Interestingly, $\mathrm{AD}$ inclusions have also been observed in the PD brain. ${ }^{72}$ In PD patients, hyperphosphorylated tau tends to aggregate in the substantia nigra in addition to other brain regions. ${ }^{80,81}$ Increased GSK-3 $\beta$ activity was correlated with the presence of hyperphosphorylated tau aggregates, suggesting that GSK-3 $\beta$ is responsible for tau phosphorylation in $\mathrm{PD}$ as in $\mathrm{AD} .^{72} \mathrm{GSK}-3 \beta$ stabilization is the gold standard for pharmacologic treatment of mood disorders. ${ }^{82}$ The decreased phosphorylation of GSK-3 $\beta$ in platelets of patients treated for depression supports the notion that GSK-3 $\beta$ contributes to the pathophysiology of depressive disorder. ${ }^{74}$ In fact, inhibiting GSK-3 $\beta$ activity was shown to impact the efficacy of antidepressant therapy. ${ }^{73,74}$ GSK-3 $\beta$ also plays a critical role in glioblastoma tumorigenesis through phosphorylation of lysine demethylase $(\mathrm{KDM}) 1 \mathrm{~A} .{ }^{83}$ Inflammation is also regulated by GSK-3 $\beta$ in neurodegenerative disorders: GSK-3 $\beta$ 

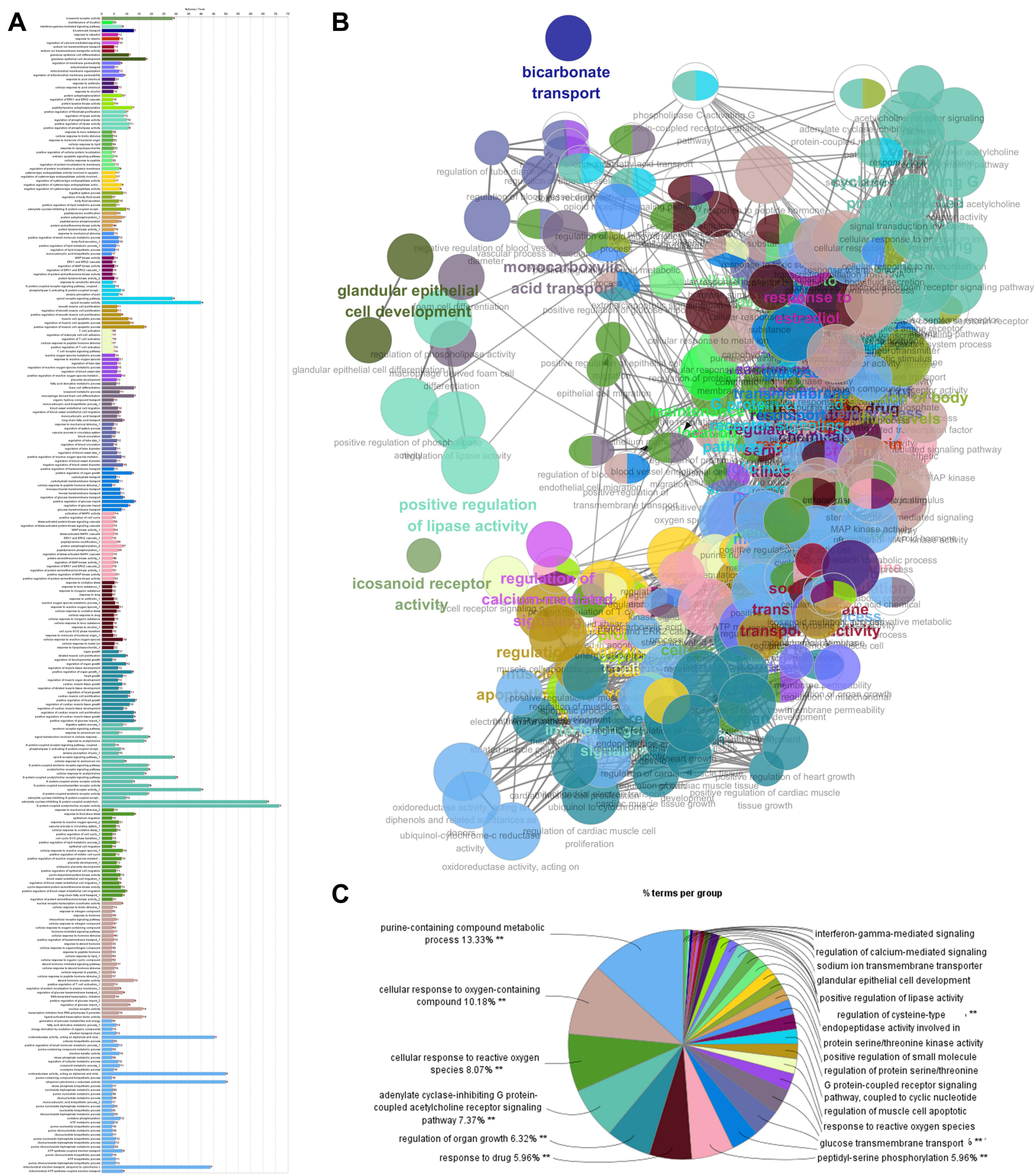

Figure 3 The target genes of icariin are mapped for the biological process terms during GO enrichment analysis by utilizing Cytoscape equipped with the ClueGO and CluePedia plugins. (A) The bars show the percentage of genes in GO terms. (B) Each node represents a GO term, and its size represents the significance. An edge indicates the existence of common genes: a finer line indicates a smaller overlap. (C) Different functional groups of GO terms were reflected by different node colors and are shown in the pie chart. $* *$ Means $p<0.01$.

overexpression stimulated the production of specific cytokines in the brain and created a proinflammatory environment that was detrimental to immature neurons. ${ }^{84-86} \mathrm{As}$ icariin inhibits GSK-3 $\beta$ in part through activation of the
PI3K/AKT signaling pathway, ${ }^{76}$ we speculate that icariin may improves $\mathrm{AD}, \mathrm{PD}$, depressive disorder, and glioblastoma by alleviating $A \beta$ pathology as well as neuroinflammation via inhibition of GSK-3 $\beta$. 


\section{Enrichment of GO Terms that Associated with Angiogenesis}

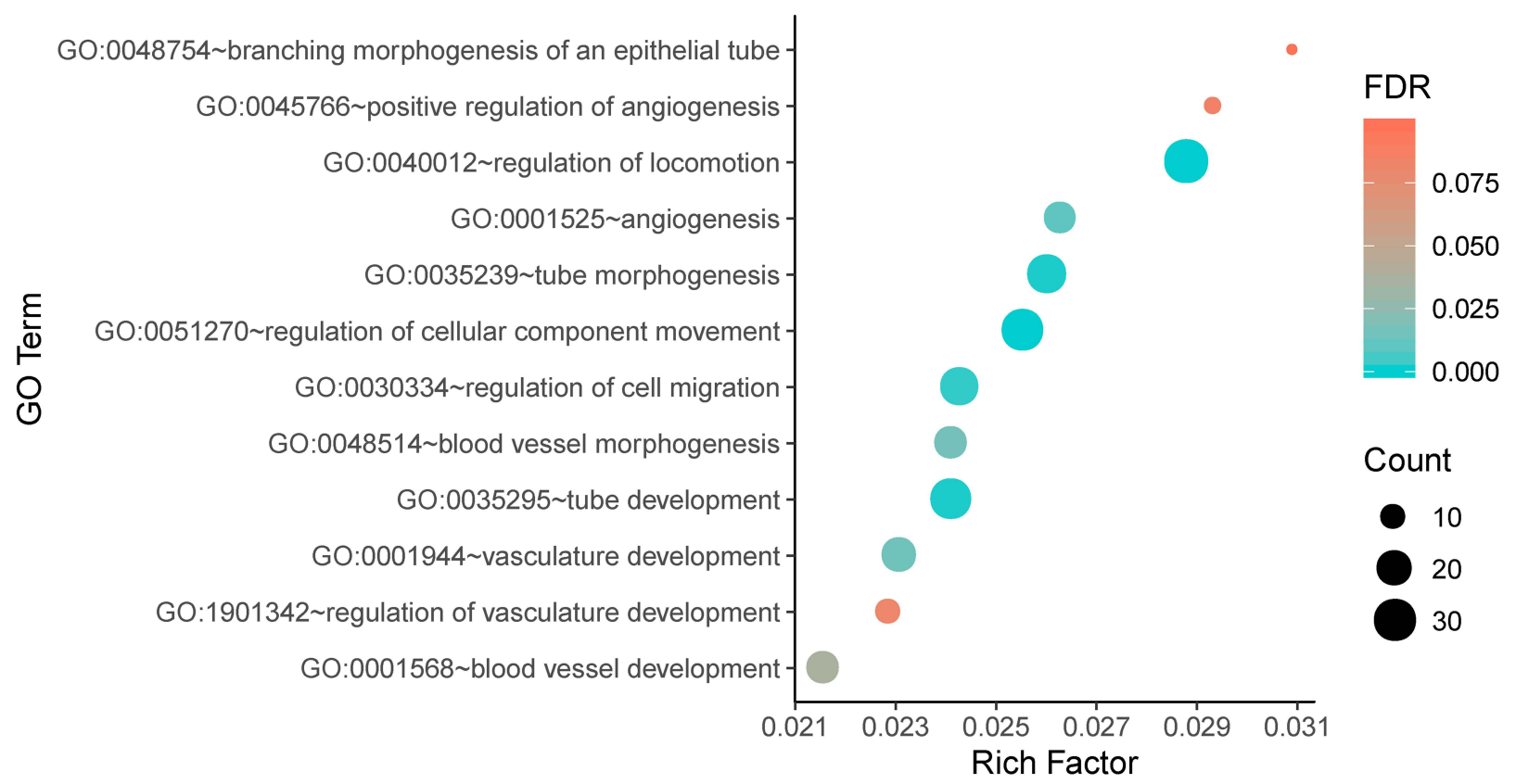

Figure 4 The bubble diagram shows significantly enriched biological process terms that pertain to angiogenesis processes. The biological process terms were shown on the $y$-axis, the rich factor of the annotated genes was presented on the x-axis, the size of term represented the gene number, and the color indicated the FDR value.

\section{$\mathrm{AD}$}

$\mathrm{AD}$ is an irreversible neurodegenerative disease that is characterized by progressive deterioration of cognitive function and memory. Various factors have been implicated in the etiology of $\mathrm{AD}$, such as abnormal protein aggregation, oxidative stress, dysregulation of calcium homeostasis, neuron and synapse degeneration, and neuroinflammation. ${ }^{76,87}$ Icariin may alleviate AD symptoms by regulating $A \beta$ production, tau phosphorylation, oxidative stress, and calcium homeostasis. ${ }^{76}$

The pathogenesis of $\mathrm{AD}$ is associated with accumulation of $A \beta$ and hyperphosphorylated tau in the brain. $A \beta$ is released from APP by BACE1, membrane-bound proteases, and $\gamma$-secretase. ${ }^{88,89}$ Icariin reduces $A \beta$ burden and deposition by inhibiting the expression of both APP and BACE1. ${ }^{89}$ Icariin counters the negative effect of $A \beta$ on synaptic plasticity via modulation of the brain-derived neurotrophic factor (BDNF)/tropomyosin receptor kinase B/AKT pathway. ${ }^{90}$ Hyperphosphorylation alters the net charge on tau protein and the conformation of the microtubule-binding region, leading to the detachment of tau from microtubules and its accumulation in neurons and aggregation as neurofibrillary tangles. ${ }^{91}$. Icariin mitigates $\mathrm{AD}$ symptoms by reducing $\mathrm{A} \beta$ and hyperphosphorylated tau levels.

Oxidative stress is also known to contribute to the pathogenesis of $\mathrm{AD} .{ }^{92}$ Icariin was shown to counteract $\mathrm{H}_{2} \mathrm{O}_{2}$-induced neurotoxicity by suppressing ROS production, and increasing the expression levels of the antioxidant enzymes catalase and peroxiredoxin (PRDX)1 via upregulation of sirtuin (SIRT) $1 .^{93}$ In primary microglia, icariin attenuated lipopolysaccharide (LPS)-induced oxidative stress and reduced ROS levels in a dose-dependent manner. ${ }^{94}$ It protected against learning and memory deficits induced by increased superoxide dismutase activity and decreased malondialdehyde level ${ }^{95}$ and abrogated the iron overload-induced Fenton reaction and oxidative stress, thereby reducing lipid peroxidation and stimulating the activities of superoxide dismutase and glutathione peroxidase. ${ }^{96}$ Thus, icariin alleviates AD symptoms by decreasing oxidative stress.

Calcium dysregulation is implicated in the progression of $\mathrm{AD}$; the failure of neurons to maintain $\mathrm{Ca}^{2+}$ homeostasis is a common feature of aging-linked neurodegenerative pathologies. ${ }^{87}$ Icariin was found to attenuate 

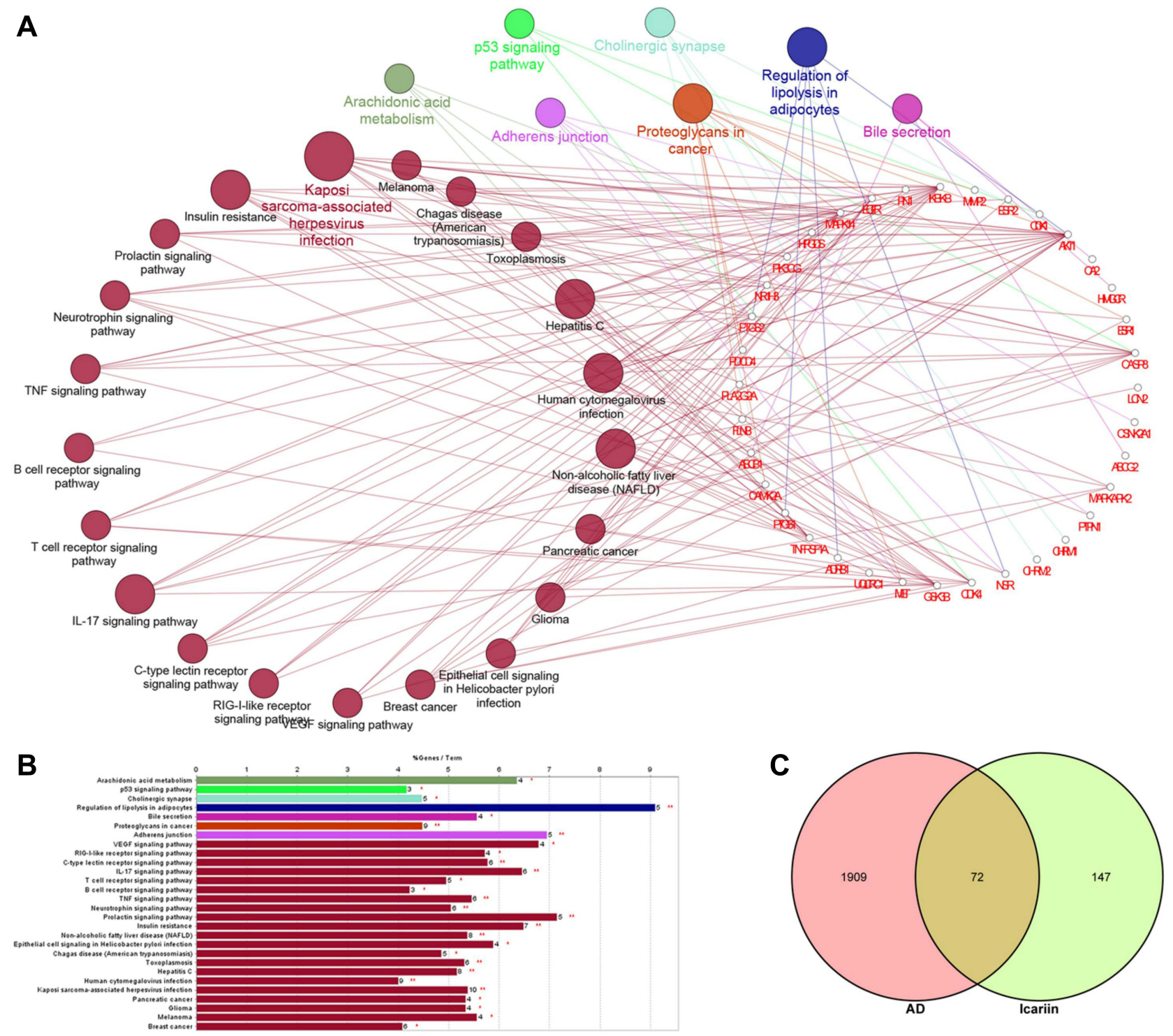

Figure 5 KEGG enrichment analysis and pathway mapping. (A) The functionally grouped network of enriched categories was generated for the target genes using ClueGO and CluePedia. Pathway terms were represented as nodes, the node colour represented the grouping information, and the node size represented the significance of the enrichment of the term. (B) The bars showed the percentage of genes in pathway terms. * means $\mathrm{p}<0.05$, $* *$ means $\mathrm{p}<0.01$. (C) The Venn diagram showed the intersection of the targets of $A D$ and icariin.

neuronal damage in a concentration-dependent manner by preventing an increase in intracellular calcium concentration. ${ }^{95}$

We identified 72 target genes common to icariin and $\mathrm{AD}$ (Figure 5C). The results of the KEGG pathway enrichment analysis indicated that icariin may mitigate $\mathrm{AD}$ by modulating the tumor necrosis factor (TNF) signaling pathway via TNF receptor (TNFR) superfamily member (TNFRSF)1A, along with mitogen-activated protein kinase (MAPK)14, and GSK-3 $\beta$ (Figure 5A and B). TNF, was linked to synaptic dysfunction in the cognitive decline associated with $\mathrm{AD} .{ }^{97}$ TNFR1, was shown to mediate $\mathrm{A} \beta$ induced neuronal cell death in $\mathrm{AD}$ and participated in amyloidogenesis by regulating BACE1, the enzyme that processes APP. ${ }^{96}$ As icariin is known to block the secretion of TNF- $\alpha,{ }^{98}$ we speculated that icariin inhibits the TNF signaling pathway and TNFRSF1A to prevent AD progression.

The autophagy-lysosome system plays an important role in the pathogenesis of AD. ${ }^{99}$ Genetic deficiency of MAPK14 stimulated autophagy, leading to reduced amyloid pathology via enhanced autophagic-lysosomal 

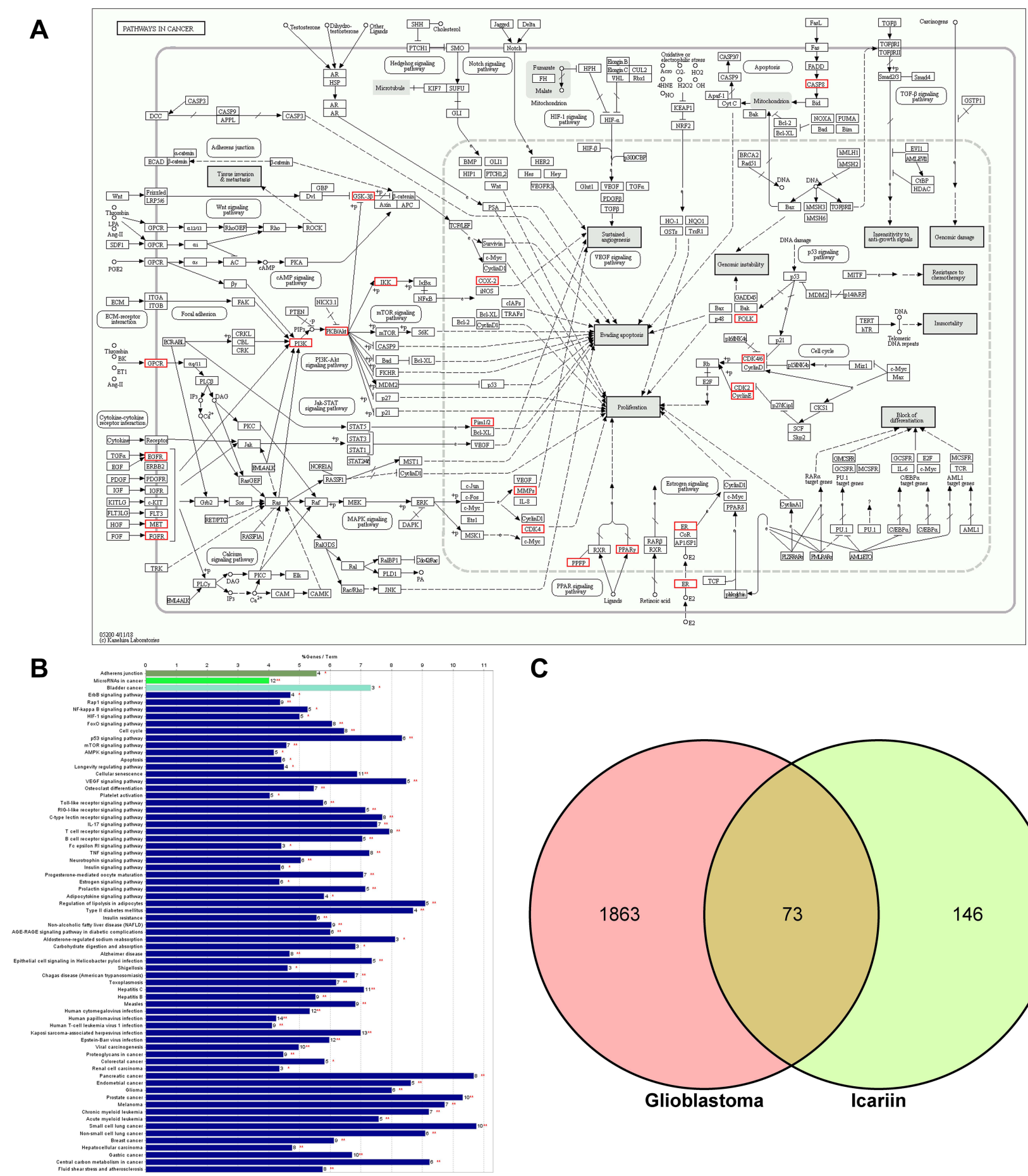

C

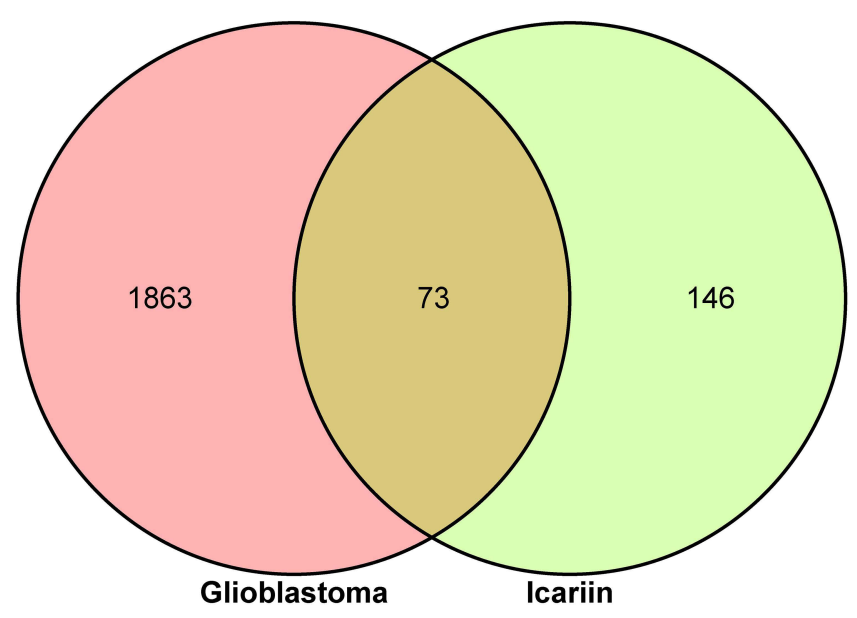

Figure 6 KEGG enrichment analysis and pathway annotation. (A) KEGG pathway map: hsa05200, Pathway in cancers, Homo sapiens (human). Red boxes marked the proteins or pathways targeted by icariin. (B) The bars showed the percentage of genes in pathway terms during KEGG enrichment analysis. $*$ Means $p<0.05$, **Means $p<0.0$ I. (C) The Venn diagram showed the intersection of the targets of glioblastoma and icariin.

degradation of BACE1. ${ }^{99}$ Thus, the suppression of MAPK14 activity is a potential therapeutic strategy to mitigate neurodegeneration in AD. Icariin was reported to inhibit the protein expression of MAPK14. ${ }^{100}$ These findings and the result of the KEGG pathway enrichment analysis suggest that icariin exerts a neuroprotective effect in $\mathrm{AD}$ by modulating the autophagy-lysosomal system through MAPK14. 


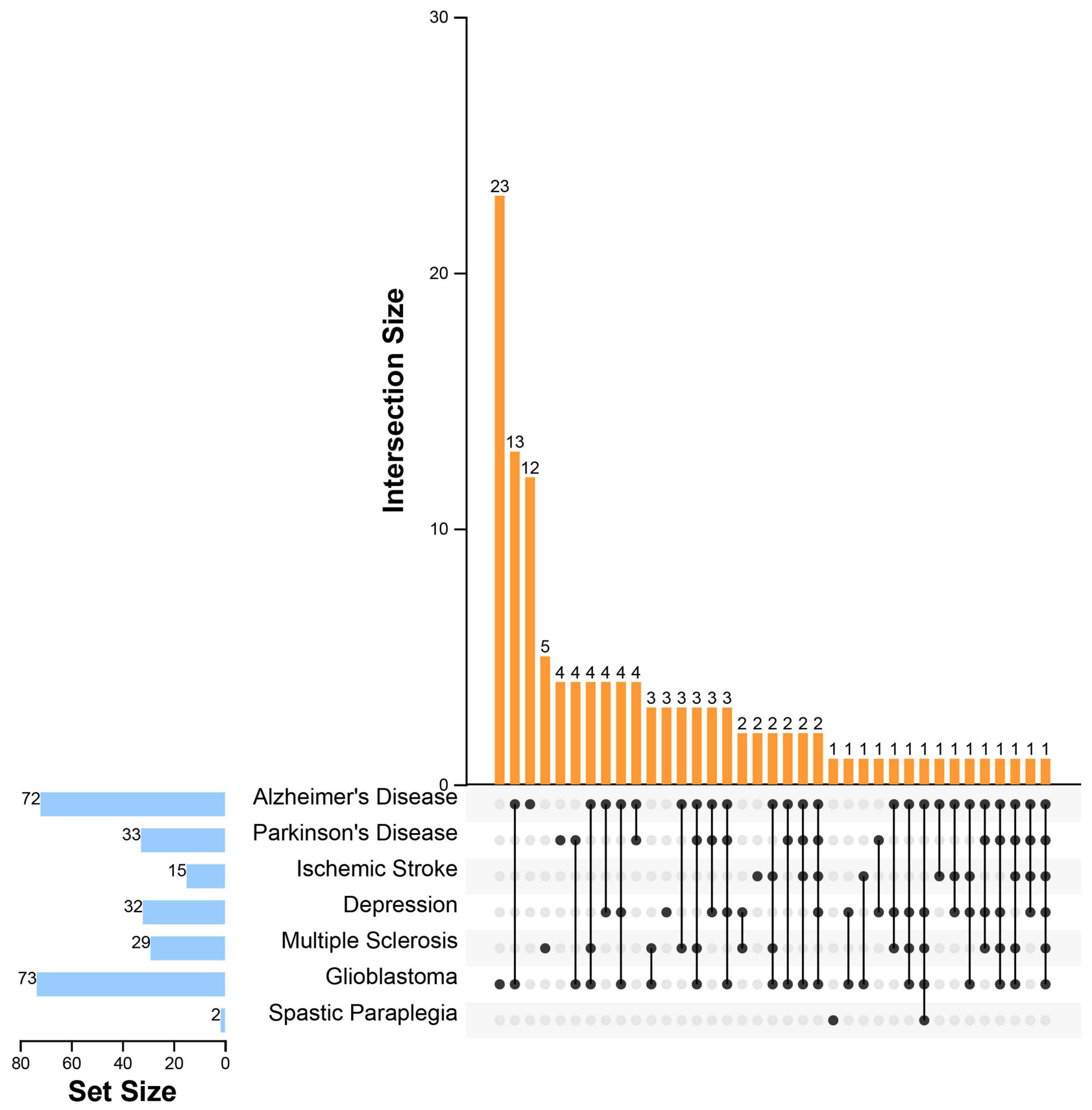

Figure 7 UpSet plot for the intersection of targets of seven nervous system diseases. The blue bar on the left of each drawing represents the amount of the targets of seven nervous system diseases, including Alzheimer's disease, Parkinson's disease, ischemic stroke, depressive disorder, multiple sclerosis, glioblastoma, and hereditary spastic paraplegias. The dark dots in the matrix at bottom part of each drawing represent the intersections of seven nervous system diseases, while the yellow bars on the top represent the target number involving in seven nervous system diseases.

\section{PD}

PD - which is characterized by motor symptoms of tremor, bradykinesia, and postural instability - is the second most common neurodegenerative disease after $\mathrm{AD} .^{101,102}$ The pathologic changes in PD are generally caused by the loss of dopaminergic neurons and depletion of dopamine pools. ${ }^{103}$
Icariin may reduce PD symptoms through multiple mechanisms. Neuroinflammation is a major pathologic event in PD. ${ }^{17} \mathrm{Nrf} 2$ is a key regulatory factor in endogenous defense systems; activation of Nrf2 signaling inhibits the production of proinflammatory factors and reduces inflammation. ${ }^{17,104}$ Microglia are brain-resident immune cells that release various proinflammatory and cytotoxic 
factors that can damage dopaminergic neurons, ultimately leading to PD. ${ }^{105}$ Icariin was found to mitigate the proinflammatory response of microglia by suppressing $\mathrm{NF}-\kappa \mathrm{B}$ signaling, thereby protecting dopaminergic neurons from damage. $^{14}$

The KEGG pathway enrichment analysis revealed multiple modules shared between icariin and PD (Supplementary Table S2). Based on the results of the network pharmacology analysis, the therapeutic effects of icariin in PD are likely associated with RAC-alpha serine/ threonine-protein kinase (AKT1), MAPK14, prostaglandin endoperoxide synthase (PTGS)2 (also known as cyclooxygenase $[\mathrm{COX}] 2)$, and GSK-3 $\beta$. PD is related to dysfunction of the nigrostriatal dopaminergic system. ${ }^{106}$ The $\mathrm{PI} 3 \mathrm{~K} / \mathrm{AKT}$ pathway modulates the antiapoptotic protein $\mathrm{B}$ cell lymphoma (Bcl)-2 in dopaminergic neurons ${ }^{107}$ activated AKT promotes the transcription and posttranscriptional activation of $\mathrm{Bcl}-2$. As AKT activation was reported to be correlated with the protective effect of icariin on the nigrostriatal system, ${ }^{107}$ icariin may target AKT/Bcl-2 activity in PD. As mentioned earlier, neuroinflammation contributes to the development and progression of PD. ${ }^{107}$ The p38 MAPK signaling pathway plays a key role in microglia activation and response that result in neuroinflammation and the degeneration of dopaminergic neurons. $^{108}$ The phosphorylation and activation of MAPK 14 lead to upregulation of PTGS2, a critical enzyme in PD pathogenesis; ${ }^{109,110}$ this induces an inflammatory response that induces dopaminergic neuron degeneration via activation of caspase 3. ${ }^{111}$ PTGS2 overexpression also stimulates the proliferation of reactive glia, which increases collagen degradation. ${ }^{109,111}$ As indicated by the results of our network pharmacology analysis, icariin inhibits MAPK14 activation and PTGS2 expression $^{14,112}$ and thus has the potential to suppress neuroinflammation and improve PD symptoms.

\section{Ischemic Stroke}

Ischemic stroke is associated with high disability and mortality and is among the leading causes of death worldwide. $^{113}$ Acute brain injury in stroke is caused by a transient restriction of the blood supply in the brain, followed by perfusion and concomitant reoxygenation. ${ }^{114}$ Icariin may exert therapeutic effects by influencing the ischemia/reperfusion process. $^{93}$

ROS generation is the initial step in brain damage after cerebral ischemia/reperfusion. ${ }^{115}$ ROS induce oxidative stress by activating several signaling pathways and react with and cause damage to lipids, proteins, and DNA. ${ }^{116,117}$ Nicotinamide adenine dinucleotide (NAD) and NAD phosphate (NADP) are essential for maintaining the intracellular balance between the generation of ROS (which ensures a reductive environment for cellular activities) and neutralization (which maintains energy homeostasis). ${ }^{118,119}$ Icariin may protect against ischemia-related brain injury by reducing oxidative stress caused by NADPH and the NADH-induced increase in ROS levels. ${ }^{120}$ Icariin was shown to inhibit NADPH oxidase (Nox) $2^{120,121}$ by decreasing the half-life of the protein, thereby suppressing ROS production to alleviate cerebrovascular smooth muscle cell hyperplasia and remodeling after ischemic stroke. ${ }^{120}$ Poststroke angiogenesis is discussed in greater detail below. The transcriptional coactivator peroxisome proliferator-activated receptor (PPAR)-gamma coactivator (PGC)-1 $\alpha$, which regulates the expression of genes involved in energy metabolism, is a key target of Nox. ${ }^{122}$ PGC- $1 \alpha$ is directly regulated by SIRT1 through phosphorylation and deacetylation; ${ }^{123}$ Meanwhile, SIRT1 is upregulated by icariin, ${ }^{93}$ which stimulates the transcriptional activity of PGC- $1 \alpha$ in neuron metabolism and mitigates mitochondrial dysfunction by inducing the upregulation of SIRT1 deacetylase. ${ }^{116}$ Additionally, icariin reduced ROS levels and brain edema following middle cerebral artery occlusion by inhibiting lactate dehydrogenase release, thereby decreasing the level of malondialdehyde and enhancing superoxide dismutase activity. ${ }^{95,124}$

Inflammation contributes to the pathophysiology of $\mathrm{AD}$, cerebral injury, cardiomyopathies, atherosclerosis, and stroke. ${ }^{125-128}$ The expression of inflammatory mediators including interleukin (IL)- $1 \beta$ and transforming growth factor (TGF)- $\beta 1$ is upregulated during ischemia. ${ }^{129} \mathrm{NF}-\kappa \mathrm{B}$ is an important transcription factor in the inflammatory response, ${ }^{130}$ PPAR $\alpha$ and PPAR $\gamma$ suppress inflammation by inhibiting the NF- $\kappa \mathrm{B}$ pathway that mediates the transcription of inflammatory mediators. ${ }^{131,132}$ In one study, icariin not only decreased the expression of IL-1 $\beta$ and TGF- $\beta 1$ in a dose-dependent manner, but also acted as an agonist of PPAR $\alpha$ and PPAR $\gamma$ and blocked NF- $\mathrm{BB}$ activation to counter the effects of ischemic stroke ${ }^{133}$ including neurologic dysfunction and infarction. ${ }^{133}$ Additionally, energy failure during ischemic stroke triggers glutamate release; ${ }^{134}$ glutamate excitotoxicity and acidosis cause brain injury through membrane receptorbased mechanisms and the resultant $\mathrm{Ca}^{2+}$ toxicity. ${ }^{135} \mathrm{As}$ a calcium channel blocker, icariin may abrogate this effect. $^{136,137}$ 
The results of the network pharmacology analysis suggested that icariin has therapeutic potential for the treatment of ischemic stroke (Supplementary Table S1). Disruption of the BBB plays a key role in the progression and exacerbation of brain injury following stroke. ${ }^{138}$ Matrix metalloproteinase (MMP)-2 mediates the degradation of tight junction proteins such as occludin and claudin-5. ${ }^{139}$ Icariin protects the BBB by reducing MMP2 protein level, ${ }^{140,141}$ a mechanism that may be relevant to the treatment of ischemic stroke. Cyclin-dependent kinase (CDK)5, is known to promote ischemic injury and strokeinduced neuronal death and potentiates the excitotoxicity caused by ischemia. ${ }^{142}$ Icariin inhibits the expression of CDK2 and CDK4; ${ }^{143}$ in our analyses, CDK5 was identified as a target of icariin, which warrants further exploration in the context of stroke treatment.

\section{Depressive Disorder}

Stress can influence the occurrence and development of depressive disorder and alter neuroendocrine function by stimulating the hypothalamic-pituitary-adrenal (HPA) axis, leading to the release of corticotropin-releasing factor (CRF) from the hypothalamus, which in turn promotes the release of adrenocorticotropin hormone and glucocorticoids such as cortisol. ${ }^{35}$ In a chronic unpredictable stress model of depression, icariin exerted an antidepressant effect by decreasing serum CRF and cortisol levels and inhibiting brain monoamine oxidase $\mathrm{A}$ and $\mathrm{B}$ activities, which increased monoamine neurotransmitter levels in brain. ${ }^{44}$ Icariin was also shown to increase glucocorticoid receptor and serotonin1A receptor (HTR1A) expression in the hippocampus and prefrontal cortex, thereby reversing chronic mild stress, ${ }^{144}$ and partly reversed the chronic mild stress-induced increases in serum and glucocorticoidinducible kinase (SGK) 1 and FK506 -binding protein (FKBP) 5 levels in the hippocampus and prefrontal cortex. ${ }^{144}$ Additionally, icariin reversed corticosteroneinduced decreases in glucose, amino acid, and lipid metabolism. ${ }^{35}$ Thus, icariin may relieve depressive disorder by reversing metabolic disturbance in the brain.

Proinflammatory cytokine levels are elevated in the brain and blood of patients with depression and aggravate depressive symptoms by increasing oxidative stress and inhibiting BDNF signaling. ${ }^{44}$ Icariin reversed the corticosterone-induced decrease in BDNF level in the hippocampus and reduced the immobility time of rats in the forced swim test $;{ }^{35}$ reduced the levels of proinflammatory factors in the hippocampus of rats under stress by inhibiting the
Nod-like receptor protein (NLRP) 3 inflammasome and caspase 1 signaling axis; ${ }^{145}$ and decreased the expression of high mobility group box (HMGB) 1 protein in the hippocampus and facilitated its translocation to the nucleus via activation of Toll-like receptor (TLR)4/ $\mathrm{X}$-box-binding protein (XBP) $1 / \mathrm{NF}-\kappa \mathrm{B}$ signaling. ${ }^{146}$

The hyperactivation of microglia is thought to play an essential role in the pathogenesis of depressive disorder. By blocking p21-activated kinase (PAK)1/ІкB kinase (IKK)/NF- $\mathrm{KB}$ and c-Jun N-terminal kinase (JNK)/p38 signaling, icariin inhibited the release and expression of proinflammatory factors such as nitric oxide, prostaglandin E-2, PTGS2, TNF- $\alpha$, IL-1 $\beta$, and IL-6 in LPS-activated microglia. ${ }^{147}$ In an in vivo study of rats treated with LPS, icariin alleviated memory and spatial learning impairments by increasing the expression of TNF- $\alpha$, IL$1 \beta$, and PTGS2 in the brain. ${ }^{148}$ These findings provide insight into the molecular mechanisms underlying the therapeutic effects of icariin in disorders associated with neuroinflammation.

Icariin may also exert antidepressant effects through the regulation of metabolism and neurotransmission. For example, icariin decreased the levels of metabotropic glutamate receptor (mGluR) 1 and mGluR 5 and increased that of excitatory amino acid transporter (EAAT) 2 in prenatal restraint stress-induced depressive disorder, ${ }^{149}$ and reversed the corticosterone-induced decreases in glucose, amino acid, and lipid metabolism. ${ }^{44}$

The antidepressant function of icariin was supported by the results of the KEGG pathway enrichment analysis of target genes common to icariin and depressive disorder, which were mainly associated with the estrogen, MAPK14, AKT1, estrogen receptor alpha and beta (ESR1 and ESR2, respectively), and GSK-3 $\beta$ signaling pathways (Supplementary Table S2). Neuronal death in the hippocampus induced by corticosterone is associated with depression; ${ }^{150}$ moreover, the activation of MAPK14 plays a critical role in corticosterone-induced apoptosis whereas PI3K/AKT signaling is linked to neuronal survival. ${ }^{151}$ The neuroprotective effect of icariin in depression may involve blocking MAPK14 and activating the PI3K/AKT pathway. ${ }^{112,152}$ ESR-signaling mediates susceptibility to depression and may impact the response to antidepressants. ${ }^{153}$ ESR1 plays an important role in depression, especially in women, and specific gene variants have been linked to severe depressive symptoms; ${ }^{154,155}$ meanwhile, ESR1 overexpression in the nucleus accumbens enhanced stress resilience in both 
sexes. ${ }^{156}$ ESR2 may promote desensitization of HTR1A signaling and thus contribute to the antidepressant response, suggesting that it could be a therapeutic target in the treatment of depressive disorder. ${ }^{154}$ Icariin activates the ESR1 signaling pathway; our network pharmacology analysis revealed that its antidepressant effect may be related to the regulation of estrogen signaling. ${ }^{155,157} \mathrm{On}$ the other hand, the decreased GSK-3 $\beta$ phosphorylation detected in the platelets of patients treated for depression implies that GSK-3 $\beta$ hyperactivation contributes to the pathophysiology of depressive disorder; this is underscored by the observation that blocking GSK-3 $\beta$ activity enhanced the effects of antidepressant therapy. ${ }^{75,158}$ Icariin was been reported to inhibit GSK-3 $\beta$ in part by activating the PI3K/AKT pathway, ${ }^{76}$ which may be the mechanism by which it alleviates depressive symptoms.

\section{MS}

MS is an autoimmune inflammatory disease that affects the CNS, with clinical manifestations such as muscle weakness, sensory deficits, cognitive impairment, and fatigue. ${ }^{159,160} \mathrm{MS}$ is thought to be caused by axon demyelination is induced by $\mathrm{T}$ cells ${ }^{161}$ and has 2 stages: early inflammation, which is responsible for relapsing-remitting disease, and delayed neurodegeneration resulting from non-relapsing progression. ${ }^{162,163}$ Icariin can potentially mitigate the symptoms of MS by suppressing immune cells: in experimental autoimmune encephalomyelitis (EAE), a widely used animal MS model, icariin decreased the pool of type 1 helper T cells (Th1) and Th17 cells and inhibited $\mathrm{T}$ cell proliferation and differentiation. ${ }^{164}$

Hyperactivation of the HPA axis has been linked to the pathogenesis of MS. ${ }^{165,166}$ An elevated corticosterone level was associated with increased inflammation in an EAE model and plaque remyelination in MS patients in clinical trials. ${ }^{166}$ In EAE, icariin was shown to modulate the HPA axis and ESR2, reduced corticosterone level, and induce the upregulation of glucocorticoid receptors in cerebral white matter. ${ }^{167}$ In another study, icariin improved EAE symptoms by decreasing corticosterone level and inhibiting inflammation and apoptosis. ${ }^{167}$ The repair of damaged myelin sheath and axons occurs during remission in the relapsing-remitting stage of $\mathrm{MS} ;{ }^{168}$ during this period, icariin accelerated myelin restoration and axon repair by stimulating neurotrophic factor production and oligodendrogenesis in the cuprizone-induced MS model. ${ }^{169}$
The network pharmacologic analysis identified annexin (ANX)A1 and histamine $\mathrm{H} 1$ receptor (H1R) as potential mediators of the therapeutic effects of icariin in MS (Supplementary Table S1). ANXA1 is an endogenous regulator of glucocorticoids that exerts anti-inflammatory effects by controlling leukocyte migration, macrophage phagocytosis, and neutrophil apoptosis. ${ }^{170}$ In a clinical trial, ANXA1 level was inversely associated with the risk of MS, and patients with lower expression of ANXA1 had more severe disabilities that was possibly due to loss of the anti-inflammatory activity of ANXA1. ${ }^{171} \mathrm{H} 1 \mathrm{R}$ is upregulated in chronic MS lesions; ${ }^{172}$ it was reported that H1R and histamine signaling increased EAE susceptibility by altering antigen-specific $\mathrm{T}$ cell effector responses, immune function, and BBB permeability. ${ }^{173}$ As pharmacologic targeting of H1R was reported to ameliorate EAE, ${ }^{174}$ we speculate that icariin inhibits $\mathrm{T}$ cell effector responses by suppressing H1R in endothelial cells. Thus, icariin may improve the symptoms of MS by targeting ANAX1 and H1R.

\section{Glioblastoma}

Icariin plays an antitumor role in many cancers including hepatocellular carcinoma, small cell lung cancer, melanoma, gastric cancer, breast cancer, and colorectal cancer. ${ }^{180-183}$ Glioblastoma multiforme (GBM) is among the most invasive, fatal, and treatment-refractory solid tumors ${ }^{184}$ and is characterized by uncontrolled proliferation, angiogenesis, and evasion of apoptosis. GBM metastasis and invasion are associated with reduced survival and poor prognosis. ${ }^{185-188}$ In our network pharmacology analysis, icariin modules were closely related to evasion of apoptosis, enhanced angiogenesis, and cell proliferation in GBM; and the results of the KEGG pathway enrichment analysis of target genes shared by icariin and glioblastoma indicated that icariin regulates the cell cycle, cellular senescence, and apoptosis, thereby contributing to the initiation, development, and progression of tumors including glioblastoma (Figure 6B).

Icariin suppresses the growth of human tumor cells by interfering with multiple signaling pathways that are critical to tumor cell growth, invasion, and apoptosis. Icariin was shown to exert antitumor effects by inducing apoptosis via the mitochondrial-mediated signaling pathway and by downregulating NF- $\mathrm{KB}$, which was accompanied by decreases in Bcl-2 and Bcl-2 extra-large (Bcl-xL) levels. ${ }^{176,189,190}$ It was also reported to inhibit the growth of cancer cells by inducing cell cycle arrest at the G2/M 
and G0/G1 phases in gallbladder carcinoma and colorectal cancer cells, respectively. Inhibiting angiogenesis can prevent tumor invasion and metastasis. ${ }^{191}$ Icariin exhibited antiangiogenic effects in xenograft models of tumors including hepatocellular carcinoma and renal cell carcinoma, ${ }^{192}$ and suppressed tumor cell migration and invasion by regulating the Rac1-dependent vasodilatorstimulated phosphoprotein (VASP) pathway. ${ }^{193}$ Icariin also enhanced the effects of antineoplastic drugs and radiation therapy; in one study, the combination of icariin and gemcitabine resulted in an enhanced antitumor effect compared to either drug alone, ${ }^{194}$ highlighting its therapeutic potential for cancer treatment.

We found that 73 target genes were shared by icariin and glioblastoma (Figure 6C). The results of the KEGG pathway enrichment analysis indicated that the antitumor functions of icariin may involve vascular endothelial growth factor (VEGF), proviral integration site for Moloney murine leukemia virus (PIM)1, CDK2, CDK4, PTGS2, epidermal growth factor receptor (EGFR), and MMP-2, among other factors (Figure 6A and B). Evasion of apoptosis is essential for tumor initiation and development; thus, inducing apoptosis is a common anticancer strategy. ${ }^{198}$ PIM1, which is overexpressed in various human cancers, modulates signaling events that promote cell growth and survival. By phosphorylating apoptosis signal-regulating kinase (ASK)1 at Ser83, PIM1 inhibited ASK1-mediated p38 kinase phosphorylation and enhanced the survival of H1299 human lung cancer cell. ${ }^{196}$ As icariin was shown to repress PIM1 expression and induce apoptosis in acute promyelocytic leukemia cell lines, ${ }^{197}$ we speculate that it can inhibit GBM progression by promoting apoptosis.

Over proliferation of tumor cells is associated with worse prognosis in GBM. ${ }^{198}$ Cyclin E2 mediates the transition from $\mathrm{G} 0 / \mathrm{G} 1$ to $\mathrm{S}$ phase via phosphorylation of the retinoblastoma $(\mathrm{Rb})$ and binding of CDK2. ${ }^{199,200}$ PIM1-mediated phosphorylation of the tyrosine phosphatase cell division cycle (CDC)25A activates CDK2/ cyclin $\mathrm{E}$ to stimulate cell proliferation by promoting cell cycle progression from G1 to S phase. ${ }^{199,200}$ CDK4 also plays important roles in the regulation of $\mathrm{G} 0 / \mathrm{G} 1$ phase and G1/S phase transition and was reported to be dysregulated in GBM. Icariin was shown to inhibit the expression of PIM1, cyclin E, CDK2, and CDK4. ${ }^{197,201}$ Based on these findings and our KEGG pathway enrichment analysis results, we propose that icariin inhibits
GBM by blocking tumor cell proliferation, possibly through negative regulation of PIM1, CDK2, and CDK4.

Angiogenesis contributes to tumor development and progression. ${ }^{186}$ As mentioned above, icariin has demonstrated antiangiogenic effect in some diseases. PTGS2 is highly expressed in diverse human cancers involving angiogenesis. Amplification of the EGFR gene is the most common genetic alteration in primary GBM, and high EGFR expression is observed in many primary human tumors. ${ }^{202}$ Hyperactivated EGFR can phosphorylate specificity protein (SP) via the p38 MAPK signaling pathway, leading to upregulation of PTGS2 and enhanced the secretion of VEGF, which stimulates angiogenesis. ${ }^{175,177}$ The inhibition of EGFR signaling and suppression of VEGF and PTGS2 by icariin also provide indirect evidence for its antiangiogenic activity. $^{203,204}$

Diffuse cell invasion is a hallmark of GBM that contributes to its lethality. Upregulation of MMP-2 was found to be correlated with higher glioma malignancy, whereas its downregulation reduced tumor invasion. ${ }^{178}$ EGFR has been shown to induce the upregulation of MMPs in the extracellular matrix, resulting in the degradation of basal membrane components and invasion of cancer cells into surrounding tissue and blood vessels. Icariin was reported to inhibit the expression of EGFR and MMP-2, ${ }^{179}$ which could prevent diffuse cell invasion in GBM based on the results of the network pharmacology analysis.

\section{Spastic Paraplegia}

Hereditary spastic paraplegias are a genetically heterogeneous group of neurodegenerative disorders characterized by length-dependent corticospinal tract and dorsal column degeneration. ${ }^{205}$ Patients present the core clinical features of bilateral lower limb spasticity, hyperreflexia, and extensor plantar responses. ${ }^{206}$ Hereditary spastic paraplegias can emerge in infancy, childhood, adolescence, or adulthood and are usually associated with autosomal dominant or recessive or X-linked modes of inheritance. ${ }^{207}$ There is currently no direct evidence that icariin has therapeutic effects in spastic paraplegia; however, a possibly link through aldehyde dehydrogenase 18 family member A1 (ALDH18A1) and EGFR was suggested by our network pharmacology analysis. ALDH18A1 mutations cause dominant $(S P G 9 A)$ or recessive $(S P G 9 B)$ spastic paraplegia. Troyer syndrome, a hereditary spastic paraplegia, is an autosomal recessive disease characterized by pathogenic mutations in the $S P G 20$ gene that result in degradation of 
the protein and a loss-of-function phenotype that includes length-dependent axonopathy of corticospinal motor neurons. $^{208}$ Methylation-induced SPG20 silencing was reported to activate the EGFR/MAPK signaling pathway. ${ }^{209}$ Spartin, a multifunctional protein encoded by SPG20, is involved in the endocytic trafficking of EGFR; ${ }^{209}$ as icariin inhibits EGFR signaling, icariin may alleviate hereditary spastic paraplegia by interfering with the function of spartin.

\section{Conclusion}

In this review, we outlined the traditional uses and chemical components of Epimedii herba and summarized the pharmacologic studies that have investigated its main active component icariin, which shows promising therapeutic effects in $\mathrm{AD}, \mathrm{PD}$, ischemic stroke, depressive disorder, MS, angiogenesis, and glioblastoma. We also used a network pharmacology approach to identify targets of icariin and performed a functional enrichment analysis to elucidate the molecular basis for the effects of icariin in these diseases. Our results along with current evidence from the literature provide a basis for future studies on the mechanisms and applications of icariin to the treatment of CNS diseases.

\section{Abbreviations}

$\mathrm{A} \beta$, amyloid beta; $\mathrm{AD}$, Alzheimer disease; AKT, protein kinase B; AKT1, RAC-alpha serine/threonine-protein kinase; ALDH18A1, aldehyde dehydrogenase 18 family member A1; ANXA1, annexin A1; APP, amyloid precursor protein; ASK1, apoptosis signal-regulating kinase 1; BACE1, $\beta$-secretase; BBB, blood-brain barrier; Bcl-2, B cell lymphoma 2; BDNF, brain-derived neurotrophic factor; CDK, cyclin-dependent kinase; CNS, central nervous system; CRF, corticotropin-releasing factor; EAE, experimental autoimmune encephalomyelitis; EGFR, epidermal growth factor receptor; ESR1, estrogen receptor alpha; ESR2, estrogen receptor beta; FDR, false discovery rate; GBM, glioblastoma multiforme; GO, Gene Ontology; GSK-3 $\beta$, glycogen synthase kinase 3 beta; H1R, histamine H1 receptor; HPA, hypothalamic-pituitary-adrenal; HTR1A, serotonin1A receptor; IL, interleukin; KEGG, Kyoto Encyclopedia of Genes and Genomes; LPS, lipopolysaccharide; MAPK, mitogen-activated protein kinase; mGluR, metabotropic glutamate receptor; MMP-2, matrix metalloproteinase 2; MS, multiple sclerosis; NADP, nicotinamide adenine dinucleotide phosphate; NF- $\mathrm{B}$, nuclear factor kappa B; NOX2, NADPH oxidase 2; Nrf2, nuclear factor erythroid 2-related factor 2; OA, oleanolic acid; PD,
Parkinson disease; PGC-1 $\alpha$, proliferator-activated receptorgamma coactivator 1 alpha; PI3K, phosphoinositide 3-kinase; PIM1, proviral integration site for Moloney murine leukemia virus 1; PPAR, peroxisome proliferator-activated receptor; PPI, protein-protein interaction; PTGS, prostaglandin endoperoxide synthase 2; ROS, reactive oxygen species; SIRT1, sirtuin 1; SPG9A, spastic paraplegia 9A, autosomal dominant; SPG9B, spastic paraplegia 9B, autosomal recessive; TGF, transforming growth factor; Th1/17 cells, type 1/ 17 helper T cells; TNF, tumor necrosis factor; TNFR1, tumor necrosis factor receptor 1; TNFRSF1A, tumor necrosis factor receptor superfamily member $1 \mathrm{~A}$; VEGF, vascular endothelial growth factor.

\section{Acknowledgments}

This work was supported by the Evidence-Based Capacity Building Project from State Administration of Traditional Chinese Medicine of the People's Republic of China (grant number: 2019XZZX-NB007), the State Administration of Traditional Chinese Medicine of the People's Republic of China (grant number: K2019BZ07), the Jiangsu Province Administration of Traditional Chinese Medicine of the People's Republic of China (grant number: ZX2016A2), and the 333 high level talents training project from Jiangsu Province Administration of Science and Technology of the People's Republic of China (grant number: BRA2016507).

\section{Author Contributions}

All authors listed on an article meet all of the following criteria. "All authors made a significant contribution to the work reported, whether that is in the conception, study design, execution, acquisition of data, analysis and interpretation, or in all these areas; took part in drafting, revising or critically reviewing the article; gave final approval of the version to be published; have agreed on the journal to which the article has been submitted; and agree to be accountable for all aspects of the work.".

\section{Disclosure}

The authors report no conflicts of interest in this work.

\section{References}

1. Feigin VL, Nichols E, Alam T. Global, regional, and national burden of neurological disorders, 1990-2016: a systematic analysis for the Global Burden of Disease Study 2016. Lancet Neurol. 2019;18 (5):459-480. doi:10.1016/S1474-4422(18)30499-X

2. Pandian JD, Gall SL, Kate MP, et al. Prevention of stroke: a global perspective. Lancet. 2018;392(10154):1269-1278. doi:10.1016/S01406736(18)31269-8 
3. Hachinski V, Einhäupl K, Ganten D, et al. Preventing dementia by preventing stroke: the Berlin Manifesto. Alzheimers Dement. 2019;15(7):961-984. doi:10.1016/j.jalz.2019.06.001

4. 2021 Alzheimer's disease facts and figures. Alzheimers Dement. 2021;17(3):327-406. doi:10.1002/alz.12328

5. Lawton MT, Vates GE. Subarachnoid Hemorrhage. $N$ Engl J Med. 2017;377(3):257-266. doi:10.1056/NEJMcp 1605827

6. Amarenco P, Kim AS. Aspirin's benefits were previously underestimated and are primarily accrued in the acute setting. Stroke. 2017;48(5):1438-1440. doi:10.1161/STROKEAHA.117.015061

7. Thelengana A, Radhakrishnan DM, Prasad M, Kumar A, Prasad K. Tenecteplase versus alteplase in acute ischemic stroke: systematic review and meta-analysis. Acta Neurol Belg. 2019;119 (3):359-367. doi:10.1007/s13760-018-0933-9

8. Li DD, Zhang YH, Zhang W, Zhao P. Meta-analysis of randomized controlled trials on the efficacy and safety of donepezil, galantamine, rivastigmine, and memantine for the treatment of Alzheimer's disease. Front Neurosci. 2019;13:472. doi:10.3389/ fnins.2019.00472

9. Mohammadi S, Jafari B, Asgharian P, Martorell M, Sharifi-Rad J. Medicinal plants used in the treatment of malaria: a key emphasis to artemisia, cinchona, cryptolepis, and tabebuia genera. Phytother Res. 2020;34(7):1556-1569. doi:10.1002/ptr.6628

10. Cho J-H, Jung J-Y, Lee B-J, Lee K, Park J-W, Bu Y. Epimedii herba: a promising herbal medicine for neuroplasticity. Phytother Res. 2017;31(6):838-848. doi:10.1002/ptr.5807

11. Wang L, Li Y, Guo Y, et al. Herba Epimedii: an ancient Chinese herbal medicine in the prevention and treatment of osteoporosis. Curr Pharm Des. 2016;22(3):328-349. doi:10.2174/ 1381612822666151112145907

12. Shen HB, Zhou YN, Zheng J, Zhu RH. ["Multi-component-multitarget-multi-pathway" mechanism of Kuihua Hugan Tablets based on network pharmacology]. Zhongguo Zhong Yao Za Zhi. 2019;44 (7):1464-1474. (Chinese). doi:10.19540/j.cnki.cjcmm.20181214.003

13. Liu $\mathrm{B}, \mathrm{Xu} \mathrm{C}, \mathrm{Wu} \mathrm{X}$, et al. Icariin exerts an antidepressant effect in an unpredictable chronic mild stress model of depression in rats and is associated with the regulation of hippocampal neuroinflammation. Neuroscience. 2015;294:193-205. doi:10.1016/j.neuroscience.2015.02.053

14. Wang G-Q, Li -D-D, Huang C, et al. Icariin reduces dopaminergic neuronal loss and microglia-mediated inflammation in vivo and in vitro. Front Mol Neurosci. 2017;10:441. doi:10.3389/ fnmol.2017.00441

15. Jin F, Gong Q-H, Xu Y-S, et al. Icariin, a phosphodiesterase-5 inhibitor, improves learning and memory in APP/PS1 transgenic mice by stimulation of NO/cGMP signalling. Int J Neuropsychopharmacol. 2014;17(6):871-881. doi:10.1017/ S1461145713001533

16. Zhang L, Shen C, Chu J, Zhang R, Li Y, Li L. Icariin decreases the expression of APP and BACE- 1 and reduces the $\beta$-amyloid burden in an APP transgenic mouse model of Alzheimer's disease. Int J Biol Sci. 2014;10(2):181-191. doi:10.7150/ijbs.6232

17. Zhang B, Wang G, He J, et al. Icariin attenuates neuroinflammation and exerts dopamine neuroprotection via an Nrf2-dependent manner. J Neuroinflammation. 2019;16(1):92. doi:10.1186/ s12974-019-1472-x

18. Chen S-J, Cui M-C. Systematic understanding of the mechanism of salvianolic acid A via computational target fishing. Molecules. 2017;22(4). doi:10.3390/molecules22040644

19. Wang Y, Ma J, Wang S, et al. Utilizing integrating network pharmacological approaches to investigate the potential mechanism of Ma Xing Shi Gan Decoction in treating COVID-19. Eur Rev Med Pharmacol Sci. 2020;24:3360-3384.

20. Li S, Zhang B. Traditional Chinese medicine network pharmacology: theory, methodology and application. Chin J Nat Med. 2013;11(2):110-120. doi:10.1016/S1875-5364(13)60037-0
21. Fuentes-Pardo AP, Ruzzante DE. Whole-genome sequencing approaches for conservation biology: advantages, limitations and practical recommendations. Mol Ecol. 2017;26(20):5369-5406. doi:10.1111/mec. 14264

22. Wang Y, Qiu J, Luo S, et al. High shear stress induces atherosclerotic vulnerable plaque formation through angiogenesis. Regen Biomater. 2016;3(4):257-267. doi:10.1093/rb/rbw021

23. Ma H, He X, Yang Y, Li M, Hao D, Jia Z. The genus Epimedium: an ethnopharmacological and phytochemical review. J Ethnopharmacol. 2011;134(3):519-541. doi:10.1016/j.jep.2011.01.001

24. Kim JY, Shim SH. Epimedium koreanum extract and its flavonoids reduced atherosclerotic risk via suppressing modification of human HDL. Nutrients. 2019;11(5):1110. doi:10.3390/nu11051110

25. Wagner H, Bauer R, Melchart D, Xiao P-G, Staudinger A. Chromatographic Fingerprint Analysis of Herbal Medicines. Springer; 2011.

26. Kang HK, Lee SB, Kwon H, Sung CK, Park YI, Dong MS. Peripubertal administration of icariin and icaritin advances pubertal development in female rats. Biomol Ther (Seoul). 2012;20 (2):189-195. doi:10.4062/biomolther.2012.20.2.189

27. Huang JM, Bao Y, Xiang W, et al. Icariin regulates the bidirectional differentiation of bone marrow mesenchymal stem cells through canonical Wnt signaling pathway. Evid Based Complement Alternat Med. 2017;2017:8085325. doi:10.1155/2017/8085325

28. Sze SCW, Tong Y, Ng TB, Cheng CLY, Cheung HP. Herba Epimedii: anti-oxidative properties and its medical implications. Molecules. 2010;15(11):7861-7870. doi:10.3390/molecules15117861

29. Ding C. [Determination of icarim in luohan jindan oral liquid by thin-layer chromatography]. Zhongguo Zhong Yao Za Zhi. 1990;15(10):604-6, 640. (Chinese).

30. Du Q, Xia M, Ito Y. Purification of icariin from the extract of Epimedium segittatum using high-speed counter-current chromatography. $J$ Chromatogr A. 2002;962(1-2):239-241. doi:10.1016/s0021-9673(02)00538-1

31. Huang RH, Zhou YC, Han W, Deng X. [Study on water extraction process of Herba epimedii with microwave technology]. Zhongguo Zhong Yao Za Zhi. 2005;30(2):107-110. (Chinese).

32. He W, Sun H, Yang B, Zhang D, Kabelitz D. Immunoregulatory effects of the herba Epimediia glycoside icariin. Arzneimittelforschung. 1995;45(8):910-913.

33. Lee MK, Choi YJ, Sung SH, Shin DI, Kim JW, Kim YC. Antihepatotoxic activity of icariin, a major constituent of Epimedium koreanum. Planta Med. 1995;61(6):523-526. doi:10.1055/s-2006-959362

34. Liu ZQ, Luo XY, Sun YX, et al. The antioxidative effect of icariin in human erythrocytes against free-radical-induced haemolysis. J Pharm Pharmacol. 2004;56(12):1557-1562. doi:10.1211/ 0022357044869

35. Gong MJ, Han B, Wang SM, Liang SW, Zou ZJ. Icariin reverses corticosterone-induced depression-like behavior, decrease in hippocampal brain-derived neurotrophic factor (BDNF) and metabolic network disturbances revealed by NMR-based metabonomics in rats. J Pharm Biomed Anal. 2016;123:63-73. doi:10.1016/j.jpba.2016.02.001

36. Kuroda M, Mimaki Y, Sashida Y, et al. Flavonol glycosides from Epimedium sagittatum and their neurite outgrowth activity on PC12h cells. Planta Med. 2000;66(6):575-577. doi:10.1055/ s-2000-8611

37. Li C, Li Q, Mei Q, Lu T. Pharmacological effects and pharmacokinetic properties of icariin, the major bioactive component in Herba Epimedii. Life Sci. 2015;126:57-68. doi:10.1016/j. Ifs. 2015.01 .006

38. Long Z, Wu J, Xiang W, Zeng Z, Yu G, Li J. Exploring the mechanism of Icariin in osteoporosis based on a network pharmacology strategy. Med Sci Monit. 2020;26:e924699. doi: $10.12659 / \mathrm{msm} .924699$ 
39. Munir N, Mahmood Z, Yameen M, Mustafa G. Therapeutic response of Epimedium gandiflorum's different doses to restore the antioxidant potential and reproductive hormones in male albino rats. Dose Response. 2020;18(3):1559325820959563. doi:10.1177/1559325820959563

40. Martens S, Mithöfer A. Flavones and flavone synthases. Phytochemistry. 2005;66(20):2399-2407. doi:10.1016/j. phytochem.2005.07.013

41. Li ZH, Ke ZC, Feng L, Jia XB. [Preparation method and pharmacological effect of baohuoside I]. Zhongguo Zhong Yao Za Zhi. 2018;43(17):3444-3450. (Chinese). doi:10.19540/j.cnki. cjcmm.20180702.007

42. Park Y, Woo SH, Seo SK, et al. Ginkgetin induces cell death in breast cancer cells via downregulation of the estrogen receptor. Oncol Lett. 2017;14(4):5027-5033. doi:10.3892/ol.2017.6742

43. Wang Z, Wang D, Yang D, Zhen W, Zhang J, Peng S. The effect of icariin on bone metabolism and its potential clinical application. Osteoporos Int. 2018;29(3):535-544. doi:10.1007/ s00198-017-4255-1

44. Jin J, Wang H, Hua X, Chen D, Huang C, Chen Z. An outline for the pharmacological effect of icariin in the nervous system. Eur J Pharmacol. 2019;842:20-32. doi:10.1016/j.ejphar.2018.10.006

45. Nicolin V, De Tommasi N, Nori SL, Costantinides F, Berton F, Di Lenarda R. Modulatory effects of plant polyphenols on bone remodeling: a prospective view from the bench to bedside. Front Endocrinol (Lausanne). 2019;10:494. doi:10.3389/fendo.2019.00494

46. Cho KS, Lim YR, Lee K, Lee J, Lee JH, Lee IS. Terpenes from forests and human health. Toxicol Res. 2017;33(2):97-106. doi:10.5487/tr.2017.33.2.097

47. Bian Q, Huang JH, Yang Z, et al. [Effects of active ingredients in three kidney-tonifying Chinese herbal drugs on gene expression profile of bone marrow stromal cells from a rat model of corticosterone-induced osteoporosis]. Zhong Xi Yi Jie he Xue Bao. 2011;9(2):179-185. (Chinese). doi:10.3736/jcim20110211

48. Shu B, Zhao Y, Wang Y, et al. Oleanolic acid enhances mesenchymal stromal cell osteogenic potential by inhibition of notch signaling. Sci Rep. 2017;7(1):7002. doi:10.1038/s41598-01707633-7

49. Cao S, Dong XL, Ho MX, et al. Oleanolic acid exerts osteoprotective effects and modulates vitamin D metabolism. Nutrients. 2018;10(2):Feb. doi:10.3390/nu10020247

50. Castellano JM, Garcia-Rodriguez S, Espinosa JM, MillanLinares MC, Rada M, Perona JS. Oleanolic acid exerts a neuroprotective effect against microglial cell activation by modulating cytokine release and antioxidant defense systems. Biomolecules. 2019;9(11):Nov. doi:10.3390/biom9110683

51. Rong ZT, Gong XJ, Sun HB, Li YM, Ji H. Protective effects of oleanolic acid on cerebral ischemic damage in vivo and $\mathrm{H}(2) \mathrm{O}$ (2)-induced injury in vitro. Pharm Biol. 2011;49(1):78-85. doi:10.3109/13880209.2010.499130

52. Zhao D, Zheng L, Qi L, et al. Structural features and potent antidepressant effects of total sterols and $\beta$-sitosterol extracted from Sargassum horneri. Mar Drugs. 2016;14(7):123. doi:10.3390/md14070123

53. Nonaka Y, Takagi T, Inai M, et al. Lauric acid stimulates ketone body production in the KT-5 astrocyte cell line. J Oleo Sci. 2016;65(8):693-699. doi:10.5650/jos.ess16069

54. Dong X, Fu J, Yin X, et al. Emodin: a review of its pharmacology, toxicity and pharmacokinetics. Phytother Res. 2016;30 (8):1207-1218. doi:10.1002/ptr.5631

55. Kukula-Koch W, Kruk-Słomka M, Stępnik K, Szalak R, Biała G. The evaluation of pro-cognitive and antiamnestic properties of berberine and magnoflorine isolated from barberry species by centrifugal partition chromatography (CPC), in relation to QSAR modelling. Int J Mol Sci. 2017;18(12):2511. doi:10.3390/ ijms 18122511
56. Atochin DN, Chernysheva GA, Smolyakova VI, et al. Neuroprotective effects of p-tyrosol after the global cerebral ischemia in rats. Phytomedicine. 2016;23(7):784-792. doi:10.1016/j.phymed.2016.03.015

57. Zhang M, Wu Y, Xie L, et al. Isoliquiritigenin protects against blood-brain barrier damage and inhibits the secretion of pro-inflammatory cytokines in mice after traumatic brain injury. Int Immunopharmacol. 2018;65:64-75. doi:10.1016/j.intimp.2018.09.046

58. Piñero J, Bravo À, Queralt-Rosinach N, et al. DisGeNET: a comprehensive platform integrating information on human disease-associated genes and variants. Nucleic Acids Res. 2017;45(D1):D833-D839. doi:10.1093/nar/gkw943

59. Zhang J, Li H, Zhang Y, Zhao C, Zhu Y, Han M. Uncovering the pharmacological mechanism of stemazole in the treatment of neurodegenerative diseases based on a network pharmacology approach. Int J Mol Sci. 2020;21(2). doi:10.3390/ijms21020427

60. UniProt Consortium T. UniProt: the universal protein knowledgebase. Nucleic Acids Res. 2018;46(5):2699. doi:10.1093/nar/gky092

61. Szklarczyk D, Gable AL, Lyon D, et al. STRING v11: protein-protein association networks with increased coverage, supporting functional discovery in genome-wide experimental datasets. Nucleic Acids Res. 2019;47(D1):D607-D613. doi:10.1093/nar/gky1131

62. Ashburner M, Ball CA, Blake JA, et al. Gene ontology: tool for the unification of biology. The Gene Ontology Consortium. Nat Genet. 2000;25(1):25-29. doi:10.1038/75556

63. Bindea G, Mlecnik B, Hackl H, et al. ClueGO: a Cytoscape plug-in to decipher functionally grouped gene ontology and pathway annotation networks. Bioinformatics (Oxford, England). 2009;25(8):1091-1093. doi:10.1093/bioinformatics/btp101

64. Mlecnik B, Galon J, Bindea G. Automated exploration of gene ontology term and pathway networks with ClueGO-REST. Bioinformatics (Oxford, England). 2019;35(19):3864-3866. doi:10.1093/bioinformatics/btz163

65. Mlecnik B, Galon J, Bindea G. Comprehensive functional analysis of large lists of genes and proteins. $J$ Proteomics. 2018;171:2-10. doi:10.1016/j.jprot.2017.03.016

66. Montañez R, Sánchez-Jiménez F, Quesada AR, Medina MÁ. Exploring and challenging the network of angiogenesis. Sci Rep. 2011;1:61. doi:10.1038/srep00061

67. Kanehisa M, Goto S. KEGG: kyoto encyclopedia of genes and genomes. Nucleic Acids Res. 2000;28(1):27-30. doi:10.1093/nar/ 28.1.27

68. Sun J, Zhang L, He Y, et al. To unveil the molecular mechanisms of Qi and blood through systems biology-based investigation into Si-Jun-Zi-Tang and Si-Wu-Tang formulae. Sci Rep. 2016;6:34328. doi:10.1038/srep34328

69. Wang Y, Fu X, Xu J, Wang Q, Kuang H. Systems pharmacology to investigate the interaction of berberine and other drugs in treating polycystic ovary syndrome. Sci Rep. 2016;6:28089. doi:10.1038/srep28089

70. Yu G, Wang L-G, Han Y, He Q-Y. clusterProfiler: an R package for comparing biological themes among gene clusters. OMICS. 2012;16(5):284-287. doi:10.1089/omi.2011.0118

71. Ritchie ME, Phipson B, Wu D, et al. Limma powers differential expression analyses for RNA-sequencing and microarray studies. Nucleic Acids Res. 2015;43(7):e47. doi:10.1093/nar/gkv007

72. Norwitz NG, Mota AS, Norwitz SG, Clarke K. Multi-loop model of Alzheimer disease: an integrated perspective on the Wnt/ GSK3 $\beta, \alpha$-Synuclein, and type 3 diabetes hypotheses. Front Aging Neurosci. 2019;11:184. doi:10.3389/fnagi.2019.00184

73. Chen J, Wang M, Waheed Khan RA, et al. The GSK3B gene confers risk for both major depressive disorder and schizophrenia in the Han Chinese population. $J$ Affect Disord. 2015;185:149-155. doi:10.1016/j.jad.2015.06.040 
74. Pláteník J, Fišar Z, Buchal R, et al. GSK3 $\beta$, CREB, and BDNF in peripheral blood of patients with Alzheimer's disease and depression. Prog Neuropsychopharmacol Biol Psychiatry. 2014;50:83-93. doi:10.1016/j.pnpbp.2013.12.001

75. Zhang Y, Huang N-Q, Yan F, et al. Diabetes mellitus and Alzheimer's disease: GSK-3 $\beta$ as a potential link. Behav Brain Res. 2018;339:57-65. doi:10.1016/j.bbr.2017.11.015

76. Angeloni C, Barbalace MC, Hrelia S. Icariin and its metabolites as potential protective phytochemicals against Alzheimer's disease. Front Pharmacol. 2019;10:271. doi:10.3389/ fphar.2019.00271

77. Cai Z, Zhao Y, Zhao B. Roles of glycogen synthase kinase 3 in Alzheimer's disease. Curr Alzheimer Res. 2012;9(7):864-879. doi:10.2174/156720512802455386

78. Llorens-Martín M, Jurado J, Hernández F, Avila J. GSK-3 $\beta$, a pivotal kinase in Alzheimer disease. Front Mol Neurosci. 2014;7:46. doi:10.3389/fnmol.2014.00046

79. Hernandez F, Lucas JJ, Avila J. GSK3 and tau: two convergence points in Alzheimer's disease. J Alzheimers Dis. 2013;33(Suppl 1):S141-S144. doi:10.3233/JAD-2012-129025

80. Yan X, Uronen RL, Huttunen HJ. The interaction of $\alpha$-synuclein and tau: a molecular conspiracy in neurodegeneration? Semin Cell Dev Biol. 2018. doi:10.1016/j.semcdb.2018.05.005

81. De Ferrari GV, Avila ME, Medina MA, Perez-Palma E, Bustos BI, Alarcon MA. Wnt/ $\beta$-catenin signaling in Alzheimer's disease. CNS Neurol Disord Drug Targets. 2014;13(5):745-754. doi:10.2174/1871527312666131223113900

82. Inkster B, Zai G, Lewis G, Miskowiak KW. GSK3ß: a plausible mechanism of cognitive and hippocampal changes induced by erythropoietin treatment in mood disorders? Transl Psychiatry. 2018;8(1):216. doi:10.1038/s41398-018-0270-z

83. Zhou A, Lin K, Zhang S, et al. Nuclear GSK3 $\beta$ promotes tumorigenesis by phosphorylating KDM1A and inducing its deubiquitylation by USP22. Nat Cell Biol. 2016;18(9):954-966. doi:10.1038/ncb3396

84. Fuster-Matanzo A, Llorens-Martín M, Sirerol-Piquer MS, García-Verdugo JM, Avila J, Hernández F. Dual effects of increased glycogen synthase kinase- $3 \beta$ activity on adult neurogenesis. Hum Mol Genet. 2013;22(7):1300-1315. doi:10.1093/ $\mathrm{hmg} / \mathrm{dds} 533$

85. Sudduth TL, Schmitt FA, Nelson PT, Wilcock DM. Neuroinflammatory phenotype in early Alzheimer's disease. Neurobiol Aging. 2013;34(4):1051-1059. doi:10.1016/j. neurobiolaging.2012.09.012

86. Llorens-Martín M, Fuster-Matanzo A, Teixeira CM, et al. GSK$3 \beta$ overexpression causes reversible alterations on postsynaptic densities and dendritic morphology of hippocampal granule neurons in vivo. Mol Psychiatry. 2013;18(4):451-460. doi:10.1038/ mp.2013.4

87. Núñez MT, Hidalgo C. Noxious iron-calcium connections in neurodegeneration. Front Neurosci. 2019;13:48. doi:10.3389/ fnins. 2019.00048

88. Jeong S. Molecular and cellular basis of neurodegeneration in Alzheimer's disease. Mol Cells. 2017;40(9):613-620. doi:10.14348/molcells.2017.0096

89. Tan JZA, Gleeson PA. The role of membrane trafficking in the processing of amyloid precursor protein and production of amyloid peptides in Alzheimer's disease. Biochim Biophys Acta Biomembr. 2019;1861(4):697-712. doi:10.1016/j. bbamem.2018.11.013

90. Sheng C, Xu P, Zhou K, Deng D, Zhang C, Wang Z. Icariin attenuates synaptic and cognitive deficits in an $A \beta(1-42)$-induced rat model of Alzheimer's disease. Biomed Res Int. 2017;2017:7464872. doi:10.1155/2017/7464872
91. Španić E, Langer Horvat L, Hof PR, Šimić G. Role of microglial cells in Alzheimer's disease tau propagation. Front Aging Neurosci. 2019;11:271. doi:10.3389/fnagi.2019.00271

92. Tönnies E, Trushina E. Oxidative stress, synaptic dysfunction, and Alzheimer's disease. $J$ Alzheimers Dis. 2017;57 (4):1105-1121. doi:10.3233/jad-161088

93. Wu B, Feng J-Y, Yu L-M, et al. Icariin protects cardiomyocytes against ischaemia/reperfusion injury by attenuating sirtuin 1-dependent mitochondrial oxidative damage. Br J Pharmacol. 2018;175(21):4137-4153. doi:10.1111/bph.14457

94. Zhou YD, Hou JG, Yang G, et al. Icariin ameliorates cisplatin-induced cytotoxicity in human embryonic kidney 293 cells by suppressing ROS-mediated PI3K/Akt pathway. Biomed Pharmacother. 2019;109:2309-2317. doi:10.1016/j.biopha.2018.11.108

95. Zheng XX, Chen YW, Yue YS, et al. Icariin ameliorates learning and memory impairments through ERK/CaMKII $\alpha / \mathrm{CREB}$ signaling and HPA axis in prenatally stressed female offspring. Biomed Pharmacother. 2019;117:109077. doi:10.1016/j. biopha.2019.109077

96. Steeland S, Gorlé N, Vandendriessche C, et al. Counteracting the effects of TNF receptor-1 has therapeutic potential in Alzheimer's disease. EMBO Mol Med. 2018;10(4):Apr. doi:10.15252/ emmm. 201708300

97. Decourt B, Lahiri DK, Sabbagh MN. Targeting tumor necrosis factor alpha for Alzheimer's disease. Curr Alzheimer Res. 2017;14(4):412-425. doi:10.2174/1567205013666160930110551

98. Fang J, Zhang Y. Icariin, an anti-atherosclerotic drug from Chinese medicinal herb horny goat weed. Front Pharmacol. 2017;8:734. doi:10.3389/fphar.2017.00734

99. Alam J, Scheper W. Targeting neuronal MAPK14/p38 a activity to modulate autophagy in the Alzheimer disease brain. Autophagy. 2016;12(12):2516-2520. doi:10.1080/ 15548627.2016 .1238555

100. Xue L, Jiang Y, Han T, et al. Comparative proteomic and metabolomic analysis reveal the antiosteoporotic molecular mechanism of icariin from Epimedium brevicornu maxim. $J$ Ethnopharmacol. 2016;192:370-381. doi:10.1016/j.jep.2016.07.037

101. Tysnes OB, Storstein A. Epidemiology of Parkinson's disease. J Neural Transm (Vienna). 2017;124(8):901-905. doi:10.1007/ s00702-017-1686-y

102. Capriotti T, Terzakis K. Parkinson disease. Home Healthc Now. 2016;34(6):300-307. doi:10.1097/nhh.0000000000000398

103. Lu DS, Chen C, Zheng YX, et al. Combination treatment of Icariin and L-DOPA against 6-OHDA-Lesioned dopamine neurotoxicity. Front Mol Neurosci. 2018;11:155. doi:10.3389/fnmol.2018.00155

104. Lee EJ, Park JS, Lee YY, Kim DY, Kang JL, Kim HS. Antiinflammatory and anti-oxidant mechanisms of an MMP-8 inhibitor in lipoteichoic acid-stimulated rat primary astrocytes: involvement of NF- $\mathrm{kB}, \mathrm{Nrf} 2$, and PPAR- $\gamma$ signaling pathways. $J$ Neuroinflammation. 2018;15(1):326. doi:10.1186/s12974-018-1363-6

105. McKenzie JA, Spielman LJ, Pointer CB, et al. Neuroinflammation as a common mechanism associated with the modifiable risk factors for Alzheimer's and Parkinson's diseases. Curr Aging Sci. 2017;10(3):158-176. doi:10.2174/ 1874609810666170315113244

106. Oh YS, Kim JS, Yoo SW, Hwang EJ, Lyoo CH, Lee KS. Striatal dopamine activity and myocardial (123) I-metaiodobenzylguanidineuptake in early Parkinson's disease. Parkinsonism Relat Disord. 2019;63:156-161. doi:10.1016/j. parkreldis.2019.02.012

107. Chen WF, Wu L, Du ZR, et al. Neuroprotective properties of icariin in MPTP-induced mouse model of Parkinson's disease: involvement of PI3K/Akt and MEK/ERK signaling pathways. Phytomedicine. 2017;25:93-99. doi:10.1016/j.phymed.2016.12.017 
108. Morganti JM, Goulding DS, Van Eldik LJ. Deletion of p38 $\alpha$ MAPK in microglia blunts trauma-induced inflammatory responses in mice. $J$ Neuroinflammation. 2019;16(1):98. doi:10.1186/s12974-019-1493-5

109. He J, Zhong W, Zhang M, Zhang R, Hu W. P38 mitogenactivated protein kinase and Parkinson's disease. Transl Neurosci. 2018;9:147-153. doi:10.1515/tnsci-2018-0022

110. Niranjan R, Mishra KP, Thakur AK. Inhibition of cyclooxygenase-2 (COX-2) initiates autophagy and potentiates MPTP-induced autophagic cell death of human neuroblastoma cells, SH-SY5Y: an inside in the pathology of Parkinson's disease. Mol Neurobiol. 2018;55(10):8038-8050. doi:10.1007/s12035-018-0950-y

111. Ge ZW, Zhu XL, Wang BC, et al. MicroRNA-26b relieves inflammatory response and myocardial remodeling of mice with myocardial infarction by suppression of MAPK pathway through binding to PTGS2. Int $J$ Cardiol. 2019;280:152-159. doi:10.1016/j.ijcard.2018.12.077

112. Liu J, Liu L, Sun J, et al. Icariin protects hippocampal neurons from endoplasmic reticulum stress and NF- $\mathrm{kB}$ mediated apoptosis in fetal rat hippocampal neurons and asthma rats. Front Pharmacol. 2019;10:1660. doi:10.3389/fphar.2019.01660

113. Putaala J. Ischemic stroke in young adults. Continuum (Minneap Minn). 2020;26(2):386-414. doi:10.1212/con.0000000000000833

114. Simon F, Oberhuber A, Floros N, et al. Acute limb Ischemia-much more than just a lack of oxygen. Int J Mol Sci. 2018;19(2):374. doi:10.3390/ijms19020374

115. Li C, Sun H, Xu G, McCarter KD, Li J, Mayhan WG. MitoTempo prevents nicotine-induced exacerbation of ischemic brain damage. J Appl Physiol. 2018;125(1):49-57. doi:10.1152/ japplphysiol.01084.2017

116. Moloney JN, Cotter TG. ROS signalling in the biology of cancer. Semin Cell Dev Biol. 2018;80:50-64. doi:10.1016/j. semcdb.2017.05.023

117. Förstermann U, Xia N, Li H. Roles of vascular oxidative stress and nitric oxide in the pathogenesis of atherosclerosis. Circ Res. 2017;120(4):713-735. doi:10.1161/circresaha.116.309326

118. Blacker TS, Duchen MR. Investigating mitochondrial redox state using NADH and NADPH autofluorescence. Free Radic Biol Med. 2016;100:53-65. doi:10.1016/j.freeradbio med.2016.08.010

119. Huang Q, Sun M, Li M, et al. Combination of $\mathrm{NAD}(+)$ and NADPH offers greater neuroprotection in ischemic stroke models by relieving metabolic stress. Mol Neurobiol. 2018;55 (7):6063-6075. doi:10.1007/s12035-017-0809-7

120. Dong H, Ming S, Fang J, Li Y, Liu L. Icariin ameliorates angiotensin II-induced cerebrovascular remodeling by inhibiting Nox2-containing NADPH oxidase activation. Hum Cell. 2019;32(1):22-30. doi:10.1007/s13577-018-0220-3

121. Belambri SA, Rolas L, Raad H, Hurtado-Nedelec M, Dang PM, El-Benna J. NADPH oxidase activation in neutrophils: role of the phosphorylation of its subunits. Eur J Clin Invest. 2018;48(Suppl 2):e12951. doi:10.1111/eci.12951

122. Tong H, Zhang X, Meng X, Lu L, Mai D, Qu S. Simvastatin inhibits activation of NADPH oxidase/p38 mapk pathway and enhances expression of antioxidant protein in Parkinson disease models. Front Mol Neurosci. 2018;11:165. doi:10.3389/ fnmol.2018.00165

123. Yang Z, Qin W, Huo J, Zhuo Q, Wang J, Wang L. MiR-22 modulates the expression of lipogenesis-related genes and promotes hepatic steatosis in vitro. FEBS Open Bio. 2021;11 (1):322-332. doi:10.1002/2211-5463.13026

124. Peng XC, Huang JR, Wang SW, et al. Traditional Chinese medicine in neuroprotection after brain insults with special reference to radioprotection. Evid Based Complement Alternat Med. 2018;2018:2767208. doi:10.1155/2018/2767208
125. Hampel H, Caraci F, Cuello AC, et al. A path toward precision medicine for neuroinflammatory mechanisms in Alzheimer's disease. Front Immunol. 2020;11:456. doi:10.3389/ fimmu.2020.00456

126. Anrather J, Iadecola C. Inflammation and stroke: an overview. Neurotherapeutics. 2016;13(4):661-670. doi:10.1007/s13311-0160483-x

127. Arida A, Protogerou AD, Kitas GD, Sfikakis PP. Systemic inflammatory response and atherosclerosis: the paradigm of chronic inflammatory rheumatic diseases. Int $J$ Mol Sci. 2018;19 (7):1890. doi:10.3390/ijms19071890

128. Li L, Zhao Q, Kong W. Extracellular matrix remodeling and cardiac fibrosis. Matrix Biol. 2018;68-69:490-506. doi:10.1016/ j.matbio.2018.01.013

129. Jia H, Ma H, Li Z, et al. Downregulation of LncRNA TUG1 inhibited TLR4 signaling pathway-mediated inflammatory damage after spinal cord ischemia reperfusion in rats via suppressing TRIL expression. J Neuropathol Exp Neurol. 2019;78 (3):268-282. doi:10.1093/jnen/nly126

130. Ji L, Du Q, Li Y, Hu W. Puerarin inhibits the inflammatory response in atherosclerosis via modulation of the NF- $\mathrm{KB}$ pathway in a rabbit model. Pharmacol Rep. 2016;68(5):1054-1059. doi:10.1016/j.pharep.2016.06.007

131. Wu JS, Tsai HD, Cheung WM, Hsu CY, Lin TN. PPAR- $\gamma$ ameliorates neuronal apoptosis and ischemic brain injury via suppressing NFкB-driven p22phox transcription. Mol Neurobiol. 2016;53 (6):3626-3645. doi:10.1007/s12035-015-9294-z

132. Su M, Cao J, Huang J, et al. The in vitro and in vivo antiinflammatory effects of a phthalimide PPAR- $\gamma$ agonist. Mar Drugs. 2017;15(1):7. doi:10.3390/md15010007

133. Xiong D, Deng Y, Huang B, et al. Icariin attenuates cerebral ischemiareperfusion injury through inhibition of inflammatory response mediated by NF-kB, PPAR $\alpha$ and PPAR $\gamma$ in rats. Int Immunopharmacol. 2016;30:157-162. doi:10.1016/j. intimp.2015.11.035

134. Yang J, Vitery MDC, Chen J, Osei-Owusu J, Chu J, Qiu Z. Glutamatereleasing SWELL1 channel in astrocytes modulates synaptic transmission and promotes brain damage in stroke. Neuron. 2019;102(4):813827.e6. doi:10.1016/j.neuron.2019.03.029

135. Belov Kirdajova D, Kriska J, Tureckova J, Anderova M. Ischemia-triggered glutamate excitotoxicity from the perspective of glial cells. Front Cell Neurosci. 2020;14:51. doi:10.3389/ fncel.2020.00051

136. Sun HS. Role of TRPM7 in cerebral ischaemia and hypoxia. $J$ Physiol. 2017;595(10):3077-3083. doi:10.1113/jp273709

137. Jiang W, Zeng M, Cao Z, et al. Icariin, a novel blocker of sodium and calcium channels, eliminates early and delayed afterdepolarizations, as well as triggered activity, in rabbit cardiomyocytes. Front Physiol. 2017;8:342. doi:10.3389/fphys.2017.00342

138. Yang C, Hawkins KE, Doré S, Candelario-Jalil E. Neuroinflammatory mechanisms of blood-brain barrier damage in ischemic stroke. Am J Physiol Cell Physiol. 2019;316(2): C135-c153. doi:10.1152/ajpcell.00136.2018

139. Li F, Geng X, Yip J, Ding Y. Therapeutic target and cell-signal communication of chlorpromazine and promethazine in attenuating blood-brain barrier disruption after ischemic stroke. Cell Transplant. 2019;28(2):145-156. doi:10.1177/0963689718819443

140. Singh WR, Devi HS, Kumawat S, et al. Angiogenic and MMPs modulatory effects of icariin improved cutaneous wound healing in rats. Eur J Pharmacol. 2019;858:172466. doi:10.1016/j. ejphar.2019.172466

141. Ren XS, Ding W, Yang XY. [Neuroprotective effect of icariin on spinal cord injury in rats]. Zhongguo Gu Shang. 2018;31 (11):1054-1060. (Chinese). doi:10.3969/j.issn.10030034.2018 .11 .014 
142. Shah K, Rossie S. Tale of the good and the Bad Cdk5: remodeling of the Actin cytoskeleton in the brain. Mol Neurobiol. 2018;55 (4):3426-3438. doi:10.1007/s12035-017-0525-3

143. Cheng X, Tan S, Duan F, Yuan Q, Li Q, Deng G. Icariin induces apoptosis by suppressing autophagy in tamoxifen-resistant breast cancer cell line MCF-7/TAM. Breast Cancer. 2019;26 (6):766-775. doi:10.1007/s12282-019-00980-5

144. Wei $\mathrm{K}, \mathrm{Xu} \mathrm{Y,} \mathrm{Zhao} \mathrm{Z,} \mathrm{et} \mathrm{al.} \mathrm{Icariin} \mathrm{alters} \mathrm{the} \mathrm{expression} \mathrm{of}$ glucocorticoid receptor, FKBP5 and SGK1 in rat brains following exposure to chronic mild stress. Int $J$ Mol Med. 2016;38 (1):337-344. doi:10.3892/ijmm.2016.2591

145. Zu Y, Mu Y, Li Q, Zhang ST, Yan HJ. Icariin alleviates osteoarthritis by inhibiting NLRP3-mediated pyroptosis. J Orthop Surg Res. 2019;14(1):307. doi:10.1186/s13018-019-1307-6

146. Liu L, Zhao Z, Lu L, et al. Icariin and icaritin ameliorated hippocampus neuroinflammation via inhibiting HMGB1-related pro-inflammatory signals in lipopolysaccharide-induced inflammation model in $\mathrm{C} 57 \mathrm{BL} / 6 \mathrm{~J}$ mice. Int Immunopharmacol. 2019;68:95-105. doi:10.1016/j.intimp.2018.12.055

147. Wang Y, Zhu T, Wang M, et al. Icariin attenuates M1 activation of microglia and $\mathrm{A} \beta$ plaque accumulation in the hippocampus and prefrontal cortex by up-regulating PPAR $\gamma$ in restraint/isolationstressed APP/PS1 mice. Front Neurosci. 2019;13:291. doi:10.3389/fnins.2019.00291

148. Shi L, Mao T, Luo P, et al. [Effect of icariin on early steroid-induced osteonecrosis of the femoral head in rabbits]. Zhongguo Xiu Fu Chong Jian Wai Ke Za Zhi. 2020;34 (2):206-212. (Chinese). doi:10.7507/1002-1892.201905112

149. Zhang X, Sun H, Su Q, et al. Antidepressant-like activity of icariin mediated by group I mGluRs in prenatally stressed offspring. Brain Dev. 2017;39(7):593-600. doi:10.1016/j. braindev.2017.03.021

150. Duman RS. Neuronal damage and protection in the pathophysiology and treatment of psychiatric illness: stress and depression. Dialogues Clin Neurosci. 2009;11(3):239-255.

151. Moosavi M, Maghsoudi N, Zahedi-Asl S, Naghdi N, Yousefpour M, Trounce IA. The role of PI3/Akt pathway in the protective effect of insulin against corticosterone cell death induction in hippocampal cell culture. Neuroendocrinology. 2008;88 (4):293-298. doi:10.1159/000150441

152. Cao LH, Qiao JY, Huang HY, et al. PI3K-AKT signaling activation and icariin: the potential effects on the perimenopausal depression-like rat model. Molecules. 2019;24(20):3700. doi: $10.3390 /$ molecules 24203700

153. Sassarini DJ. Depression in midlife women. Maturitas. 2016;94:149-154. doi:10.1016/j.maturitas.2016.09.004

154. Kajta M, Wnuk A, Rzemieniec J, et al. Depressive-like effect of prenatal exposure to DDT involves global DNA hypomethylation and impairment of GPER1/ESR1 protein levels but not ESR2 and AHR/ARNT signaling. $J$ Steroid Biochem Mol Biol. 2017;171:94-109. doi:10.1016/j.jsbmb.2017.03.001

155. Lin HY, Tsai JC, Wu LY, Peng WH. Reveals of new candidate active components in hemerocallis radix and its anti-depression action of mechanism based on network pharmacology approach Int J Mol Sci. 2020;21(5). doi:10.3390/ijms21051868

156. Lorsch ZS, Loh YE, Purushothaman I, et al. Estrogen receptor $\alpha$ drives pro-resilient transcription in mouse models of depression. Nat Commun. 2018;9(1):1116. doi:10.1038/s41467-018-03567-4

157. Li X, Peng B, Pan Y, et al. Icariin stimulates osteogenic differentiation and suppresses adipogenic differentiation of rBMSCs via estrogen receptor signaling. Mol Med Rep. 2018;18 (3):3483-3489. doi:10.3892/mmr.2018.9325
158. Jiang Y, Zhang Y, Su L. MiR-539-5p decreases amyloid $\beta$-protein production, hyperphosphorylation of tau and memory impairment by regulating PI3K/Akt/GSK-3 $\beta$ pathways in APP/PS1 double transgenic mice. Neurotox Res. 2020;38(2):524-535. doi:10.1007/s12640-020-00217-w

159. Multiple sclerosis. Nat Rev Dis Primers. 2018;4(1):44. doi:10.1038/s41572-018-0046-z

160. Owens B. Multiple sclerosis. Nature. 2016;540(7631):S1. doi: $10.1038 / 540$ S1a

161. Danikowski KM, Jayaraman S, Prabhakar BS. Regulatory T cells in multiple sclerosis and myasthenia gravis. $J$ Neuroinflammation. 2017;14(1):117. doi:10.1186/s12974-017-0892-8

162. Steinman L, Zamvil SS. Beginning of the end of two-stage theory purporting that inflammation then degeneration explains pathogenesis of progressive multiple sclerosis. Curr Opin Neurol. 2016;29(3):340-344. doi:10.1097/wco.0000000000000317

163. Rüther BJ, Scheld M, Dreymueller D, et al. Combination of cuprizone and experimental autoimmune encephalomyelitis to study inflammatory brain lesion formation and progression. Glia. 2017;65(12):1900-1913. doi:10.1002/glia.23202

164. Hao H, Zhang Q, Zhu H, et al. Icaritin promotes tumor T-cell infiltration and induces antitumor immunity in mice. Eur J Immunol. 2019;49(12):2235-2244. doi:10.1002/eji.201948225

165. Akcali A, Zengin F, Aksoy SN, Zengin O. Fatigue in multiple Sclerosis: is it related to cytokines and hypothalamic-pituitaryadrenal axis? Mult Scler Relat Disord. 2017;15:37-41. doi:10.1016/j.msard.2017.03.004

166. Dos Santos N, Novaes LS, Dragunas G, et al. High dose of dexamethasone protects against EAE-induced motor deficits but impairs learning/memory in C57BL/6 mice. Sci Rep. 2019;9 (1):6673. doi:10.1038/s41598-019-43217-3

167. Wei Z, Wang $M$, Hong $M$, et al. Icariin exerts estrogen-like activity in ameliorating EAE via mediating estrogen receptor $\beta$, modulating HPA function and glucocorticoid receptor expression. Am J Transl Res. 2016;8(4):1910-1918

168. Lassmann H. Pathogenic mechanisms associated with different clinical courses of multiple sclerosis. Front Immunol. 2018;9:3116. doi:10.3389/fimmu.2018.03116

169. Zhang $\mathrm{Y}$, Yin $\mathrm{L}$, Zheng N, et al. Icariin enhances remyelination process after acute demyelination induced by cuprizone exposure. Brain Res Bull. 2017;130:180-187. doi:10.1016/j.brainresbull.2017.01.025

170. Han PF, Che XD, Li HZ, Gao YY, Wei XC, Li PC. Annexin A1 involved in the regulation of inflammation and cell signaling pathways. Chin J Traumatol. 2020;23(2):96-101. doi:10.1016/j. cjtee.2020.02.002

171. Colamatteo A, Maggioli E, Azevedo Loiola R, et al. Reduced annexin A1 expression associates with disease severity and inflammation in multiple sclerosis patients. $J$ Immunol. 2019;203(7):1753-1765. doi:10.4049/jimmunol.1801683

172. Rafiee Zadeh A, Falahatian M, Alsahebfosoul F. Serum levels of histamine and diamine oxidase in multiple sclerosis. Am J Clin Exp Immunol. 2018;7(6):100-105.

173. Kaskow BJ, Baecher-Allan C. Effector T cells in multiple sclerosis. Cold Spring Harb Perspect Med. 2018;8(4):a029025. doi:10.1101/cshperspect.a029025

174. Thangam EB, Jemima EA, Singh H, et al. The role of histamine and histamine receptors in mast cell-mediated allergy and inflammation: the Hunt for new therapeutic targets. Front Immunol. 2018;9:1873. doi:10.3389/fimmu.2018.01873

175. Liu Z, Wang J, Huang X, Li Z, Liu P. Deletion of sirtuin 6 accelerates endothelial dysfunction and atherosclerosis in apolipoprotein E-deficient mice. Transl Res. 2016;172:18-29.e2. doi:10.1016/j.trsl.2016.02.005 
176. Chen $\mathrm{M}, \mathrm{Wu}$ J, Luo Q, et al. The anticancer properties of herba epimedii and its main bioactive componentsicariin and Icariside II. Nutrients. 2016;8(9):Sep. doi:10.3390/nu8090563

177. Liu Y, Zhang H, Yan L, et al. MMP-2 and MMP-9 contribute to the angiogenic effect produced by hypoxia/15-HETE in pulmonary endothelial cells. J Mol Cell Cardiol. 2018;121:36-50. doi:10.1016/j.yjmcc.2018.06.006

178. Zhang H, Ma Y, Wang H, Xu L, Yu Y. MMP-2 expression and correlation with pathology and MRI of glioma. Oncol Lett. 2019;17(2):1826-1832. doi:10.3892/ol.2018.9806

179. Wang P, Zhang F, He Q, et al. Flavonoid compound Icariin activates hypoxia inducible factor- $1 \alpha$ in chondrocytes and promotes articular cartilage repair. PLoS One. 2016;11(2):e0148372e0148372. doi:10.1371/journal.pone.0148372

180. Wu X, Kong W, Qi X, et al. Icariin induces apoptosis of human lung adenocarcinoma cells by activating the mitochondrial apoptotic pathway. Life Sci. 2019;239:116879. doi:10.1016/j. lfs.2019.116879

181. Li X, Sun J, Hu S, Liu J. Icariin induced B16 melanoma tumor cells apoptosis, suppressed tumor growth and metastasis. Iran J Public Health. 2014;43(6):847-848.

182. Sun ZG, Lang ZF, Mu YD, et al. Therapeutic effect and mechanism of icariin combined with calcium sensitive receptor on mouse gastric cancer cells. J Biol Regul Homeost Agents. 2020;34 (5):1831-1836. doi:10.23812/20-228-1

183. Shen R, Wang JH. The effect of icariin on immunity and its potential application. Am J Clin Exp Immunol. 2018;7(3):50-56.

184. Yang L, Wang Y, Guo H, Guo M. Synergistic anti-cancer effects of icariin and temozolomide in glioblastoma. Cell Biochem Biophys. 2015;71(3):1379-1385. doi:10.1007/s12013-0140360-3

185. Monteiro AR, Hill R, Pilkington GJ, Madureira PA. The role of hypoxia in glioblastoma invasion. Cells. 2017;6(4):45. doi:10.3390/cells6040045

186. Yang C, Zheng J, Xue Y, et al. The effect of MCM3AP-AS1/miR211/KLF5/AGGF1 axis regulating glioblastoma angiogenesis. Front Mol Neurosci. 2017;10:437. doi:10.3389/fnmol.2017.00437

187. Gao YF, Zhu T, Mao XY, et al. Silencing of Forkhead box D1 inhibits proliferation and migration in glioma cells. Oncol Rep. 2017;37(2):1196-1202. doi:10.3892/or.2017.5344

188. Ma C, Wei F, Xia $\mathrm{H}$, et al. MicroRNA-10b mediates TGF$\beta 1$-regulated glioblastoma proliferation, migration and epithelialmesenchymal transition. Int J Oncol. 2017;50(5):1739-1748. doi:10.3892/ijo.2017.3947

189. Xu B, Jiang $\mathrm{C}$, Han $\mathrm{H}$, et al. Icaritin inhibits the invasion and epithelial-to-mesenchymal transition of glioblastoma cells by targeting EMMPRIN via PTEN/AKt/HIF-1 $\alpha$ signalling. Clin Exp Pharmacol Physiol. 2015;42(12):1296-1307. doi:10.1111/14401681.12488

190. Shi DB, Li XX, Zheng HT, et al. Icariin-mediated inhibition of $\mathrm{NF}-\kappa \mathrm{B}$ activity enhances the in vitro and in vivo antitumour effect of 5-fluorouracil in colorectal cancer. Cell Biochem Biophys. 2014;69(3):523-530. doi:10.1007/s12013-014-9827-5

191. Tan HL, Chan KG, Pusparajah P, Lee LH, Goh BH. Gynura procumbens: an overview of the biological activities. Front Pharmacol. 2016;7:52. doi:10.3389/fphar.2016.00052

192. Tan HL, Chan KG, Pusparajah P, et al. Anti-cancer properties of the naturally occurring aphrodisiacs: Icariin and its derivatives. Front Pharmacol. 2016;7:191. doi:10.3389/fphar.2016.00191

193. Zheng CM, Liu XZ, Li QL, Wang JF, Tan Z, Ge MH. [The bisphenol A-enhanced activity of thyroid carcinoma cell line B-CPAP is inhibited by Icarrin]. Zhonghua Er Bi Yan Hou Tou Jing Wai Ke Za Zhi. 2017;52(6):458-462. (Chinese). doi:10.3760/ cma.j.issn.1673-0860.2017.06.012
194. Lei K, Ma B, Shi P, et al. Icariin mitigates the growth and invasion ability of human oral squamous cell carcinoma via inhibiting toll-like receptor 4 and phosphorylation of NF- $\kappa \mathrm{B}$ P65. Onco Targets Ther. 2020;13:299-307. doi:10.2147/ott. $\mathrm{S} 214514$

195. Guo J, Qu H, Chen Y, Xia J. The role of RNA-binding protein tristetraprolin in cancer and immunity. Med Oncol. 2017;34 (12):196. doi:10.1007/s12032-017-1055-6

196. Zhu HH, Wang XT, Sun YH, et al. Pim1 overexpression prevents apoptosis in cardiomyocytes after exposure to hypoxia and oxidative stress via upregulating cell autophagy. Cell Physiol Biochem. 2018;49(6):2138-2150. doi:10.1159/000493817

197. Zhang H, Li P, Li J, et al. Icariin induces apoptosis in acute promyelocytic leukemia by targeting PIM1. Pharmacol Rep. 2017;69(6):1270-1281. doi:10.1016/j.pharep.2017.06.005

198. Liang P, Chai Y, Zhao H, Wang G. Predictive analyses of prognostic-related immune genes and immune infiltrates for glioblastoma. Diagnostics (Basel). 2020;10(3). doi:10.3390/ diagnostics10030177

199. Wang S, Wang K, Deng Z, et al. Correlation between the protein expression levels of A-kinase anchor protein95, p-retinoblastoma (Ser780), cyclin D2/3, and cyclin E2 in esophageal cancer tissues. Asia Pac J Clin Oncol. 2019;15(5):e162-e166. doi:10.1111/ ajco. 13146

200. Cayrol F, Praditsuktavorn P, Fernando TM, et al. THZ1 targeting CDK7 suppresses STAT transcriptional activity and sensitizes T-cell lymphomas to BCL2 inhibitors. Nat Commun. 2017;8:14290. doi:10.1038/ncomms 14290

201. Wang $P$, Zhang J, Xiong X, et al. Icariin suppresses cell cycle transition and cell migration in ovarian cancer cells. Oncol Rep. 2019;41(4):2321-2328. doi:10.3892/or.2019.6986

202. Hatanpaa KJ, Burma S, Zhao D, Habib AA. Epidermal growth factor receptor in glioma: signal transduction, neuropathology, imaging, and radioresistance. Neoplasia. 2010;12(9):675-684. doi:10.1593/neo.10688

203. Hua $\mathrm{W}$, Zhang $\mathrm{Y}, \mathrm{Wu} \mathrm{X}$, et al. Icariin attenuates interleukin-1 $\beta$ induced inflammatory response in human nucleus pulposus cells. Curr Pharm Des. 2018;23(39):6071-6078. doi:10.2174/ 1381612823666170615112158

204. Algandaby MM, Breikaa RM, Eid BG, Neamatallah TA, AbdelNaim AB, Ashour OM. Icariin protects against thioacetamide-induced liver fibrosis in rats: implication of anti-angiogenic and anti-autophagic properties. Pharmacol Rep. 2017;69(4):616-624. doi:10.1016/j.pharep.2017.02.016

205. Shribman S, Reid E, Crosby AH, Houlden H, Warner TT. Hereditary spastic paraplegia: from diagnosis to emerging therapeutic approaches. Lancet Neurol. 2019;18(12):1136-1146. doi:10.1016/s1474-4422(19)30235-2

206. Boutry M, Morais S, Stevanin G. Update on the genetics of spastic paraplegias. Curr Neurol Neurosci Rep. 2019;19(4):18. doi:10.1007/s11910-019-0930-2

207. de Souza PVS, de Rezende Pinto WBV, de Rezende Batistella GN, Bortholin T, Oliveira ASB. Hereditary spastic paraplegia: clinical and genetic hallmarks. Cerebellum. 2017;16 (2):525-551. doi:10.1007/s12311-016-0803-z

208. Liang H, Miao H, Yang H, et al. Dwarfism in Troyer syndrome: a family with SPG20 compound heterozygous mutations and a literature review. Ann N Y Acad Sci. 2020;1462(1):118-127. doi:10.1111/nyas.14229

209. Zhou Z, Wang W, Xie X, Song Y, Dang C, Zhang H. Methylationinduced silencing of SPG20 facilitates gastric cancer cell proliferation by activating the EGFR/MAPK pathway. Biochem Biophys Res Commun. 2018;500(2):411-417. doi:10.1016/j. bbrc.2018.04.089 


\section{Publish your work in this journal}

Drug Design, Development and Therapy is an international, peerreviewed open-access journal that spans the spectrum of drug design and development through to clinical applications. Clinical outcomes, patient safety, and programs for the development and effective, safe, and sustained use of medicines are a feature of the journal, which has also been accepted for indexing on PubMed Central. The manuscript management system is completely online and includes a very quick and fair peer-review system, which is all easy to use. Visit http://www. dovepress.com/testimonials.php to read real quotes from published authors.

Submit your manuscript here: https://www.dovepress.com/drug-design-development-and-therapy-journal 